\title{
Coupling impedances of a gap in vacuum chamber
}

\author{
Yoshihiro Shobuda \\ JAEA, Japan Atomic Energy Agency, 2-4 Shirakata Shirane, Tokaimura, Nakagun, Ibaraki 319-1195, Japan \\ Yong Ho Chin and Koji Takata \\ KEK, High Energy Accelerator Research Organization, 1-1 Oho, Tsukuba, Ibaraki 305-0801, Japan
}

(Received 21 November 2006; published 24 April 2007)

\begin{abstract}
A gap in the vacuum chamber stands between a beam and the outside world, and the theoretical elucidation of the interaction mechanism between the gap and the beam is of great importance to understand the interaction of any device with the beam. In this paper, we will present the formulas for the longitudinal and transverse impedances due to a gap in the beam chamber. In this process, we will derive the complete solutions of electromagnetic fields effective in the entire region, including the inside and the outside of the chamber, in a form that they can be easily numerically evaluated. The newly developed technique can provide new methods of solutions of electromagnetic fields also for a rather broad class of structures such as cavities. The numerical results of impedances are consistent with the ABCI results and their behavior in high frequency agrees well with the prediction of the diffraction theory. Our theory can also accurately reproduce the behavior of the impedance near and above the cutoff frequencies. In addition, our theory is applicable even to the impedances for nonrelativistic beams. We found that the broadband impedance of the small cavitylike structure can be estimated from the gap size and the chamber radius only, regardless of the exact shape of the structure. We also found that the transverse impedance of a gap has a large resonance peak at the frequency where the wavelength is equal to the chamber circumference. This resonance peak appears around 1-2 GHz in most of the cases, and we should be careful to design a ceramic break so that this transverse mode will not leak out to interact with nearby devices.
\end{abstract}

DOI: 10.1103/PhysRevSTAB.10.044403

\section{INTRODUCTION}

When a beam circulates inside the vacuum chamber in a ring, it can see the outside world of the chamber only through "windows" in the chamber. These windows can be either gaps or holes (holes are mostly for pumping purposes, and thus we will omit holes from our considerations hereafter). In other words, if one or any devise interacts with a beam, they can do it only through gaps in the chamber. The outside of the gap can be open such as in a ceramic break (in this case, the gap is filled with ceramic to shield the vacuum) [1] or housed by metal such as in a cavity. In any case, gaps play fundamental roles as a mediator in interaction between a beam and the outside world of the vacuum chamber. The theoretical elucidation of the interaction mechanism in the gap is of great importance to understand interaction of any device with the beam.

A beam circulating inside the vacuum chamber is accompanied with the image current running on the surface of the chamber. When it crosses a gap, the image current has to also jump to the other side of the gap, becoming the displacement current. Since the beam current has AC components, this $\mathrm{AC}$ displacement current between the gap creates the radiation field (in other words, the gap works like a slot antenna). The beam receives the deceleration field as the backreaction of this radiation field. This deceleration mechanism can be formulated as resistive
PACS numbers: 29.20.Lq, 41.20.-q, 02.30.-f, 41.75.-i

impedance to the beam. The gap in the chamber also creates capacitive impedance like a parallel-plate capacitor.

Despite its great importance in the foundation of the accelerator theory, only few works have been done for analytical understanding of the interaction between a gap and a beam. Nevertheless, some sketchy information can be obtained from previous works in the accelerator theory or the antenna theory. Papas already found the analytical formula for radiation far from the gap when an $\mathrm{AC}$ voltage is applied to the gap in the chamber in his antenna theory in 1949 [2]. This information gives us some hints to find out the resistive impedance of the gap. On the other hand, the imaginary part of admittance is intuitively obtained from the equivalent electric circuit theory. For the high frequency region, there is another approach based on diffraction theory [3]. This theory predicts that the longitudinal impedance damps as $k^{-1 / 2}$ [3]. In fact, we succeeded to create a somehow patchy theory from this information and found that its calculation accuracy of gap impedance is as good as the present theory for most of the cases. However, we found finally that we can get complete solutions of electromagnetic fields in every place either near or far from the gap, and thus formulated the interaction between the gap and the beam in a systematic and comprehensive fashion. The analytical results can be summarized in the form of coupling impedance and can be directly compared with the numerical results of $\mathrm{ABCI}$ code [4]. 
It has been believed that a gap in the chamber or a ceramic break will increase the longitudinal impedance, but not the transverse one [5]. This is because "the differential currents can avoid the high impedance region," namely, the transverse image current can run within one chamber, without crossing to the chamber of the other side. It has been also believed that "the transverse impedance is increased only at very high frequency," "around the pipe cutoff frequency, typically between 1 and $2 \mathrm{GHz}$ " [5]. On the other hand, the diffraction theory predicts that the transverse impedance decreases in proportion to $k^{-3 / 2}$ [3]. As we will see, our analytical results show that this conjecture is partially true, though the transverse impedance decreases at very high frequency (except at the cutoff frequency where impedance increases slightly). Actually, we found that there is a large resonance peak at the frequency where the wavelength is equal to the chamber circumference.

As stated previously, the way that the electromagnetic fields are created by the image current (induced by a beam) running across the gap is similar to that of the radiation field generated by the AC voltage applied between the gap in a slot antenna. The property of this radiation field far from the gap was analyzed by Silver and Saunders in the antenna theory [6]. The field matching method can easily derive the formal solutions of the radiation fields outside of the chamber. However, these solutions include integrals of complicated combination of the Hankel functions with full of singular points (cuts and poles) and can be hardly evaluated numerically. They used the saddle point method to evaluate these integrals and derived a simple approximation only good for the far-zone fields. They considered that it is a "task of considerable magnitude" to derive a near field solution, and since then this problem has been left unsolved. That may be acceptable in the antenna theory where only the radiation field in the far zone is of great interest. Obviously, to evaluate the coupling impedance in the accelerator theory, we need to derive the complete solutions effective in the entire region, including the inside of the chamber. As a matter of fact, we succeeded to derive such solutions suitable for numerical evaluations as will be seen in this paper and verified the validity of the use of the saddle point method by Silver and Saunders. Our method is applicable to any speed of a beam: not limited to the ultrarelativistic beam.

When a bunch passes through a cavitylike structure (with a small gap and a surrounding outside structure), it will interact with the gap first, and then the fields created at the gap will be radiated to the inside of the structure. If a bunch is short enough (typically shorter than the chamber radius), it will not have enough time to see the details of the structure, even vaguely in one passage. Though the whole structure will determine the exact shape of the impedance, its short-range impact to the beam will be dominated by the two parameters: the gap size and the chamber radius. In other words, the short-range wake potentials or the broadband impedance of a cavitylike structure are almost determined by these two parameters. The loss factor is a good measure to find out the broadband impedance and its frequency dependency. If our conjecture is true, the loss factor of a small cavitylike structure can be estimated once the gap size is given, in addition to the chamber radius and the bunch length. This sounds reasonable, since the loss of beam energy should be equal to the radiated energy from the gap, which is determined by the gap size. We will investigate this conjecture in the coming session.

In this paper, we study the system in which a beam with any speed passes through a small gap, by comparison with the radius of the metal chambers. The impedance is calculated by a semianalytical approach using the field matching technique [7]. We explain how to carry out the integration of the formula for impedances, where the Hankel functions impose a task of considerable magnitude on evaluation of the integrals. We derive the longitudinal impedance in Sec. II. We will show that the gap size, the chamber radius, and the bunch length determine the loss factor of a small cavity in a good accuracy. We also discuss the accuracy of the saddle point analysis of the radiation field. In Sec. III, the properties of transverse impedance are investigated. The paper is concluded in Sec. IV.

\section{A FORMULA FOR LONGITUDINAL IMPEDANCE WITH SPACE CHARGE EFFECTS}

Let us start with deriving electromagnetic fields generated by the interaction between a beam and a gap in the cylindrically symmetric system. We use the cylindrical coordinates $(\rho, \theta, z)$ as shown in Fig. 1. We assume that the thin metal chamber has the radius of $a$, and the gap is located in the region where $-w<z<w$ (namely, the width of the gap is $2 w$ ).

In order to obtain formal solutions of the fields, we apply the field matching technique to this system. We assume that the beam has the cylindrically uniform density with the radius of $\sigma$ and its total charge is $1 \mathrm{C}$. Namely, its current density is given by

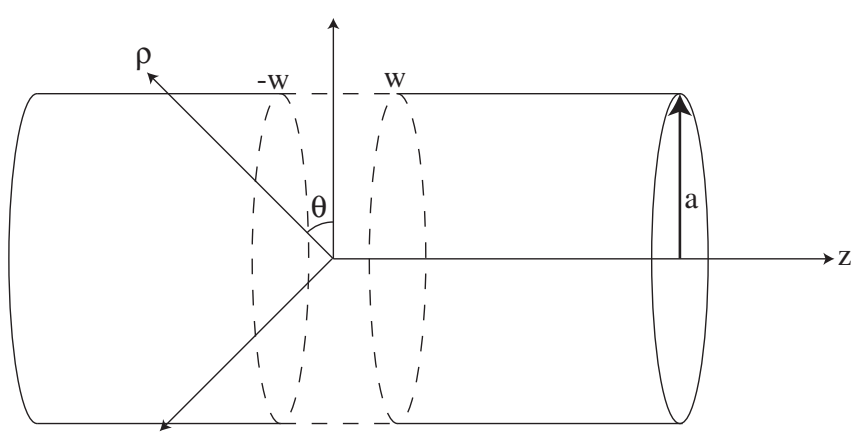

FIG. 1. The gap in the thin metal chamber and the cylindrical coordinate system $(\rho, \theta, z)$. 


$$
j_{z}=\beta c[1-\Theta(\rho-\sigma)] e^{-j k z+j \omega t} /\left(\pi \sigma^{2}\right),
$$

where $\Theta(x)$ is the step function. The formal solutions of the fields at the frequency $\omega$ inside the chamber are given by (see Appendices A and B)

$$
\begin{aligned}
E_{z}= & \frac{j k}{\gamma^{2}} \frac{c Z_{0}}{\pi \sigma^{2}}\left(\frac{1}{\bar{k}^{2}}-\frac{\sigma I_{0}(\bar{k} \rho) K_{1}(\bar{k} \sigma)}{\bar{k}}\right) e^{-j k z} \\
+ & \int_{-\infty}^{\infty} d h A(h) e^{-j h z} \frac{J_{0}(\Lambda \rho)}{J_{0}(\Lambda a)} \\
H_{\theta}= & \frac{\beta c}{\pi \sigma} K_{1}(\bar{k} \sigma) I_{1}(\bar{k} \rho) e^{-j k z}+\frac{j k \beta}{Z_{0}} \\
& \times \int_{-\infty}^{\infty} d h A(h) e^{-j h z} \frac{J_{1}(\Lambda \rho)}{\Lambda J_{0}(\Lambda a)}
\end{aligned}
$$

for $\rho<\sigma$, and

$$
\begin{aligned}
E_{z}= & \frac{j c Z_{0}}{\pi \sigma \gamma} I_{1}(\bar{k} \sigma) K_{0}(\bar{k} \rho) e^{-j k z} \\
& +\int_{-\infty}^{\infty} d h A(h) e^{-j h z} \frac{J_{0}(\Lambda \rho)}{J_{0}(\Lambda a)}, \\
H_{\theta}= & \frac{\beta c}{\pi \sigma} I_{1}(\bar{k} \sigma) K_{1}(\bar{k} \rho) e^{-j k z}+\frac{j k \beta}{Z_{0}} \\
& \times \int_{-\infty}^{\infty} d h A(h) e^{-j h z} \frac{J_{1}(\Lambda \rho)}{\Lambda J_{0}(\Lambda a)},
\end{aligned}
$$

for $\rho>\sigma$, where $A(h)$ is the expansion coefficient, $I_{m}(z), K_{m}(z)$ are the modified Bessel functions, $J_{m}(z)$ is the Bessel function, $\Lambda=\sqrt{k^{2} \beta^{2}-h^{2}}, k=2 \pi f / c \beta$, and $\bar{k}=k / \gamma$. The time dependence of the fields is assumed to be harmonic and it is expressed as the complex exponential $e^{j \omega t}$.

In order to apply the field matching technique [7], we have to know the fields outside the chamber. Silver and Saunders have already derived such fields at $\rho>a$ (see Appendix C) [6]. Following their theory, we describe the fields as follows:

$$
\begin{gathered}
E_{z}=\frac{\tilde{V}}{2 \pi} \int_{-\infty}^{\infty} \frac{H_{0}^{(2)}(\Lambda \rho)}{H_{0}^{(2)}(\Lambda a)} \frac{\sin h w}{h w} e^{-j h z} d h, \\
H_{\theta}=j \frac{\beta k}{Z_{0}} \frac{\tilde{V}}{2 \pi} \int_{-\infty}^{\infty} \frac{H_{1}^{(2)}(\Lambda \rho)}{\Lambda H_{0}^{(2)}(\Lambda a)} \frac{\sin h w}{h w} e^{-j h z} d h,
\end{gathered}
$$

where $H_{m}^{(2)}(z)$ is the Hankel functions of the second kind. Here $\tilde{V}$ is the voltage between the gap and will be determined by the boundary condition.

Since the fields inside the chamber [given by Eqs. (4) and (5)] and outside the chamber [given by Eqs. (6) and (7)] must be continuous at $\rho=a$, the gap voltage $\tilde{V}$ can be solved as follows:

$$
\tilde{V}=\frac{4 w \gamma c Z_{0} I_{1}(\bar{k} \sigma) e^{-j k z}}{j k^{2} \sigma a I_{0}(\bar{k} a) \int d h \int_{-w}^{w} d \xi \frac{e^{-j h(z-\xi)}}{\Lambda}\left(\frac{H_{1}^{(2)}(\Lambda a)}{H_{0}^{(2)}(\Lambda a)}-\frac{J_{1}(\Lambda a)}{J_{0}(\Lambda a)}\right)},
$$

where $z$ is the matching point for the magnetic fields inside and outside the chamber. The average value of $E_{z}$ [expressed by Eq. (2) over $\rho$ ] gives the coupling impedance (see Appendix B):

$$
\begin{gathered}
Z_{\|}=-\frac{j Z_{0}}{k \beta \pi \sigma^{2}}\left(1-\frac{2 K_{0}(\bar{k} a) I_{1}^{2}(\bar{k} \sigma)}{I_{0}(\bar{k} a)}-2 I_{1}(\bar{k} \sigma) K_{1}(\bar{k} \sigma)\right) \mathcal{C}+Z_{\text {gap }, \|}, \\
Z_{\text {gap }, \|}=\frac{8 w Z_{0} I_{1}^{2}(\bar{k} \sigma) e^{-j k z}}{j \beta \gamma \sigma^{2} a \bar{k}^{3} I_{0}^{2}(\bar{k} a) \int_{-w}^{w} d \xi \int d h \frac{e^{-j h(z-\xi)}}{\Lambda}\left(\frac{J_{1}(\Lambda a)}{J_{0}(\Lambda a)}-\frac{H_{1}^{(2)}(\Lambda a)}{H_{0}^{(2)}(\Lambda a)}\right)},
\end{gathered}
$$

where $\mathcal{C}$ is the circumference of the accelerator ring. The first term in Eq. (9) represents the nonrelativistic space charge impedance (see Appendix B) [8]. The second term $Z_{\text {gap, } \|}$ in Eq. (9) is the coupling impedance of the gap.

The integration of the Bessel functions can be done simply by picking up residues in the complex plane $h$. However, the integration of the Hankel functions is a quite complicated and difficult task since there are branch points at $h= \pm k \beta$ in the complex plane $h$. To proceed further, we first rewrite the integration of the Hankel functions as shown in Eq. (D3). This manipulation enables the integra- tion of the Hankel functions over $h$ with the usual residue theorem. We choose the path of integration to be below the poles for $h<0$ and above the poles for $h>0$. Finally, we obtain the expression of the longitudinal coupling impedance:

$$
Z_{\text {gap, }, \|}=\frac{4 Z_{0} I_{1}^{2}(\bar{k} \sigma) e^{-j k z}}{j \beta \gamma \sigma^{2} a \bar{k}^{3} I_{0}^{2}(\bar{k} a)\left(Y_{\text {pole }}+Y_{\text {cut }}\right)}
$$

where 


$$
\begin{gathered}
Y_{\text {pole }}=-\sum_{s=1}^{\infty} \frac{\pi a\left(2-e^{-j\left(b_{s} / a\right)(z+w)}-e^{-j\left(b_{s} / a\right)(z-w)}\right)}{w b_{s}^{2}} \\
Y_{\text {cut }}=-\frac{1}{w \pi a} \int_{0}^{\infty} d \zeta \frac{2-e^{-j(z+w) \sqrt{k^{2} \beta^{2}+\left(\zeta / a^{2}\right)}}-e^{j(z-w) \sqrt{k^{2} \beta^{2}+\left(\zeta / a^{2}\right)}}}{\zeta\left(k^{2} \beta^{2}+\frac{\zeta}{a^{2}}\right) H_{0}^{(1)}\left(e^{j(\pi / 2)} \sqrt{\zeta}\right) H_{0}^{(2)}\left(e^{j(\pi / 2)} \sqrt{\zeta}\right)} \simeq \frac{2(1-j)}{\sqrt{k \beta w}} .
\end{gathered}
$$

Here $b_{s}^{2}=k^{2} \beta^{2} a^{2}-j_{0, s}^{2}=-\beta_{s}^{2}, j_{0, s}$ are $s$ th zeros of $J_{0}(z)$, and $H_{m}^{(1)}(z)$ is the Hankel function of the first kind. We should notice that $b_{s}$ approaches $-j \beta_{s}$ for $j_{0, s}>k \beta a$.

In the above derivation, we used Eqs. (D1) and (D3) of Appendix D. The integration in Eq. (13) over $\zeta$ is much more straightforward than the integration in Eq. (10) over $h$, since there is no singular point except $\zeta=0$ along the integration path.

\section{A. Comparison with ABCI results}

Now, let us check the accuracy of Eq. (11) for the gap impedance by comparing with the $\mathrm{ABCI}$ result. As a typical set of parameters, we choose the chamber radius $a=$ $50 \mathrm{~mm}$, the half width of gap $w=4 \mathrm{~mm}$, and the beam radius $\sigma=5 \mathrm{~mm}$. In $\mathrm{ABCI}$, we put a huge cavity in the outside of the gap to simulate the open space. Figure 2 shows the comparison results of the real (left) and imaginary (right) parts of the impedance, respectively. Quite good agreements can be seen. Both results show that the impedance decreases in proportion to $k^{-1 / 2}$ in high frequency, as predicted by the diffraction theory. In low frequency, there is a little discrepancy between $\mathrm{ABCI}$ and Eq. (11). This discrepancy comes from our approximation of the gap voltage $\tilde{V}$ as a constant. It is interesting to point out that the real part of impedance has a peak at zero frequency: namely, there is more radiation at lower frequency. The imaginary part of the impedance is purely capacitive, and has a sharp peak near zero frequency and vanishes at zero frequency.

The amazing feature of the present theory is that it can accurately reproduce the behavior of the impedance near the cutoff frequency, which can be identified as a dip and a small bump near $2.3 \mathrm{GHz}$ in Fig. 2. Below the cutoff frequency, the fields created at the gap can radiate away only to the outside of the chamber. The fields radiated to the inside of the chamber simply decay. Exactly at the cutoff frequencies, the fields inside the chamber can spread out to the infinity and stay there without propagating away. In other words, the capacitance of the gap becomes infinite and can store any amount of radiation energy inside. Thus, no radiation comes out of the gap and the impedance vanishes. Once the frequency goes beyond the cutoff frequency, the propagation of the radiation fields to the inside of the chamber is permitted in addition to the outside. This new channel of the radiation stimulates more radiation from the gap and thus the impedance increases.

\section{B. Consistency with the previous studies}

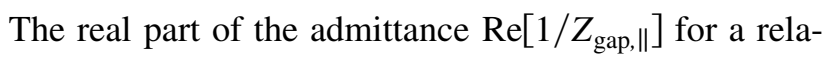
tivistic beam should be equal to the radiation conductance
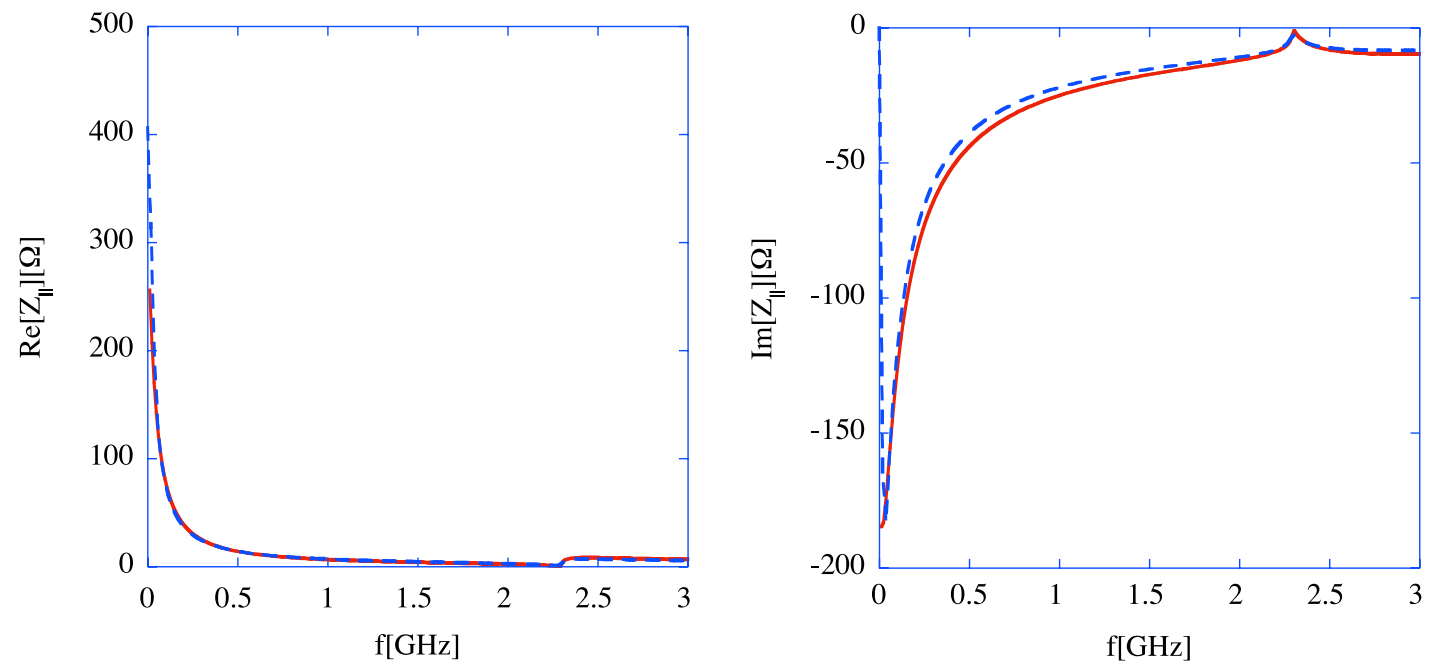

FIG. 2. (Color) Comparison of the real (left) and imaginary (right) parts of the longitudinal impedance, calculated by the present theory (red curves) and ABCI (blue curves), respectively. The parameters are as follows: $\gamma=1000, a=5 \mathrm{~cm}, w=4 \mathrm{~mm}, z=0 \mathrm{~m}$, and $\sigma=a / 10$. 


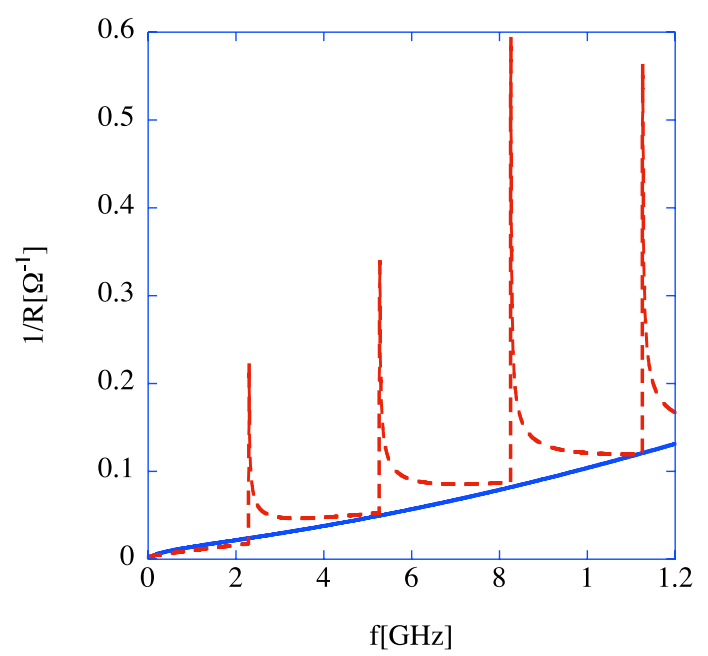

FIG. 3. (Color) Comparison of the admittance, calculated by Eq. (11) (red curve) and Eqs. (15) and (16) (blue curve), respectively.

of the slot antenna found by Papas. The general expression of the radiation conductance $1 / R$ is given by

$$
\frac{1}{R}=\frac{1}{60 \pi^{2}} \int_{-\pi / 2}^{\pi / 2} \frac{d \theta}{\cos \theta H_{0}^{(1)}(k a \cos \theta) H_{0}^{(1)}(k a \cos \theta)^{*}} .
$$

For the low frequency region $(k a<1)$, Papas simplified this expression to

$$
\frac{1}{R}=\frac{1}{120|\log k a|}
$$

For $k a>0.1$, the empirical formula can be found from the numerical fitting of the integration in Eq. (14):

$$
\begin{aligned}
\frac{1}{R}= & \frac{k a}{60 \pi^{2}} \times 1.12 \times[7.17-3.03 \log k a \\
& \left.+0.9393(\log k a)^{2}\right] .
\end{aligned}
$$

Figure 3 shows the comparison of the real part of the admittances given by our Eq. (11) and by Eqs. (15) and (16). They are in a good agreement except near the cutoff frequencies. The sharp rises of the real part of the admittances in our theory represent the new channel of the radiation to the inside of the chamber, while only the radiation to the outside of the chamber is considered in Papas' theory.

\section{The longitudinal impedance for a nonrelativistic beam}

Our formalism can also deal with the longitudinal impedance for a nonrelativistic beam. We expect that the impedance for a nonrelativistic beam is smaller than that for a relativistic beam, since the slow beam will receive more kicks from the fluctuating rf fields while going through the cloud of the radiation fields. Figure 4 shows the dependence of longitudinal impedance on Lorentz factor $\gamma$. In this example, we assume the chamber with the large radius $a=250 \mathrm{~mm}$, because the $\gamma$-dependence of longitudinal impedance is more pronounced for a larger $a$. We can see from this figure that the impedance for a nonrelativistic beam with $\gamma$ of 1.2 is indeed smaller than for a relativistic beam with $\gamma$ larger than several. It can be also identified that the modulation of impedance around the cutoff frequencies is more enhanced for a larger $\gamma$.
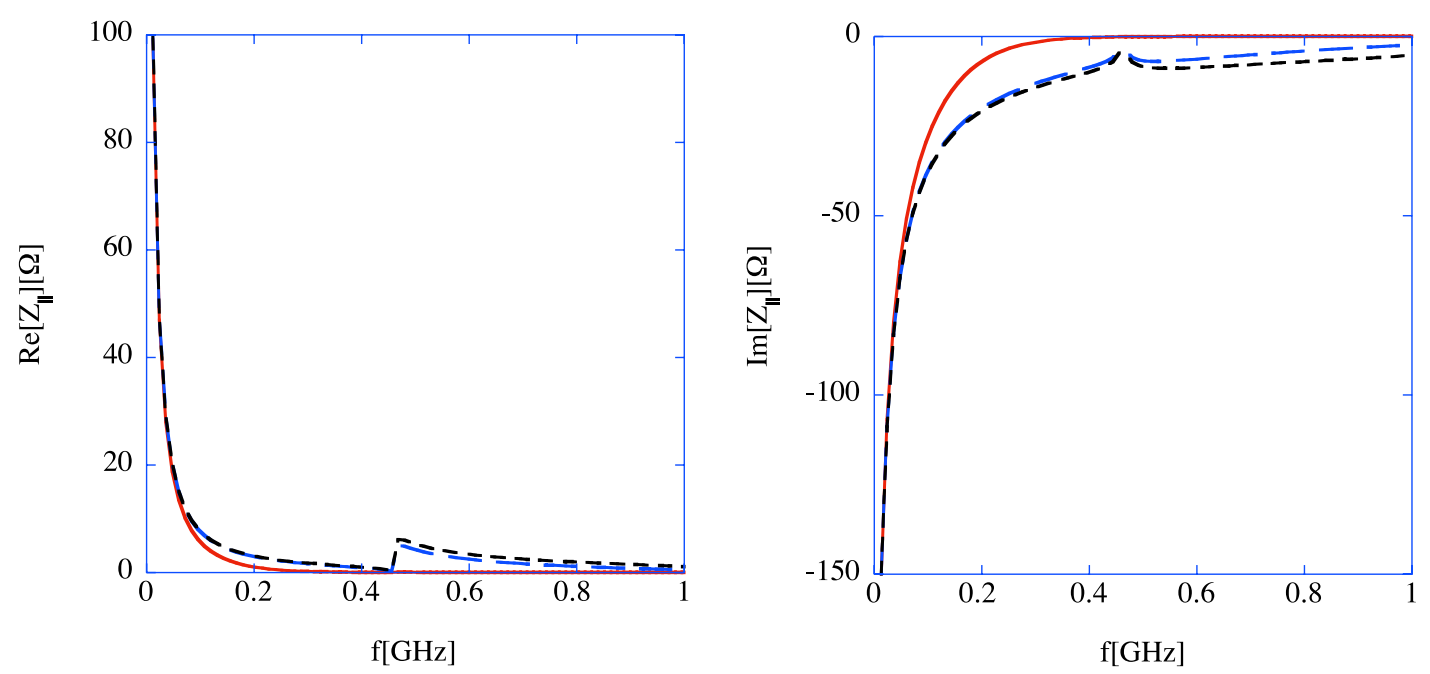

FIG. 4. (Color) The dependence of longitudinal impedance on Lorentz factor $\gamma$. We assume the chamber with the large radius of $a=250 \mathrm{~mm}$. The red, blue, and black curves represent $\gamma=1.2, \gamma=4$, and $\gamma=1000$ cases, respectively. The left and the right figures show the real and the imaginary parts of longitudinal impedance, respectively. The impedance for a nonrelativistic beam with $\gamma$ of 1.2 is indeed smaller than for a relativistic beam with gamma larger than several. 


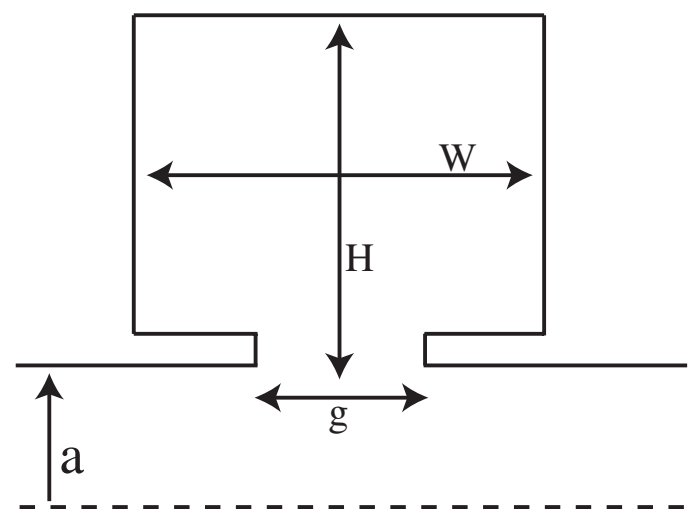

FIG. 5. The cavitylike structure parametrized by its width $W$, its height $H$, and its opening size $g$ (the gap size).

\section{The loss factor}

As stated in the Introduction, we expect that the gap will play a dominant role in the short-range impact of a small cavitylike structure to a short bunch in one passage. Even though the detail of the impedance depends on the exact shape of the structure, its broadband impedance can be approximately estimated once the gap size and the chamber radius are known. The loss factor for an arbitrary bunch length will be a good measure to evaluate this conjecture numerically.

The loss factor $\kappa$ is determined by the real part of impedance. The loss factor for a Gaussian distribution with the rms length of $\sigma_{s}$, or $\sigma_{t}=\sigma / \beta c$ in time can be calculated from

$$
\kappa=\int_{0}^{\infty} d f 2 \operatorname{Re}\left[Z_{\|}(f)\right] e^{-4 \pi^{2} f^{2} \sigma_{t}^{2}}
$$

We consider the cavitylike structure parametrized by its width $W$, its height $H$, and its opening size $g$ (the gap size), as shown in Fig. 5. For simplicity, we pick up the case that $W$ is equal to $H$ and change $W=H$ sides for various chamber radii $a$ and gap sizes $g$, to see how quickly the loss factor saturates as $W=H$ increases. Figure 6 shows the results of this exercise. The vertical axis represents the ratio of the loss factor calculated by numerical calculation to that by the present theory [obtained from Eq. (10)], and the horizontal axis shows the value of $W=H$. We can see that the loss factor of the cavity saturates very quickly to that of the gap as the cavity size increases, though the saturated ratio is not exactly one due to the small discrepancy between the numerical and theoretical impedance at low frequency. Roughly speaking, the loss factor of the cavity saturates to that of the gap when the size of the cavity $W=H$ exceeds twice the size of the gap. This exercise demonstrates that the loss factor of a cavitylike structure can be estimated in a good accuracy from the gap size, the chamber radius, and the bunch length, regardless of the exact shape of the structure.

\section{E. The validity of the saddle point method for the calculation of radiation fields}

Silver and Saunders used the saddle point method to simplify the formal solutions of the radiated field with a full of singular points due to the Hankel functions, for the far-zone fields in their antenna theory. Since there is a cut in the complex plane of integration, there is some doubt if
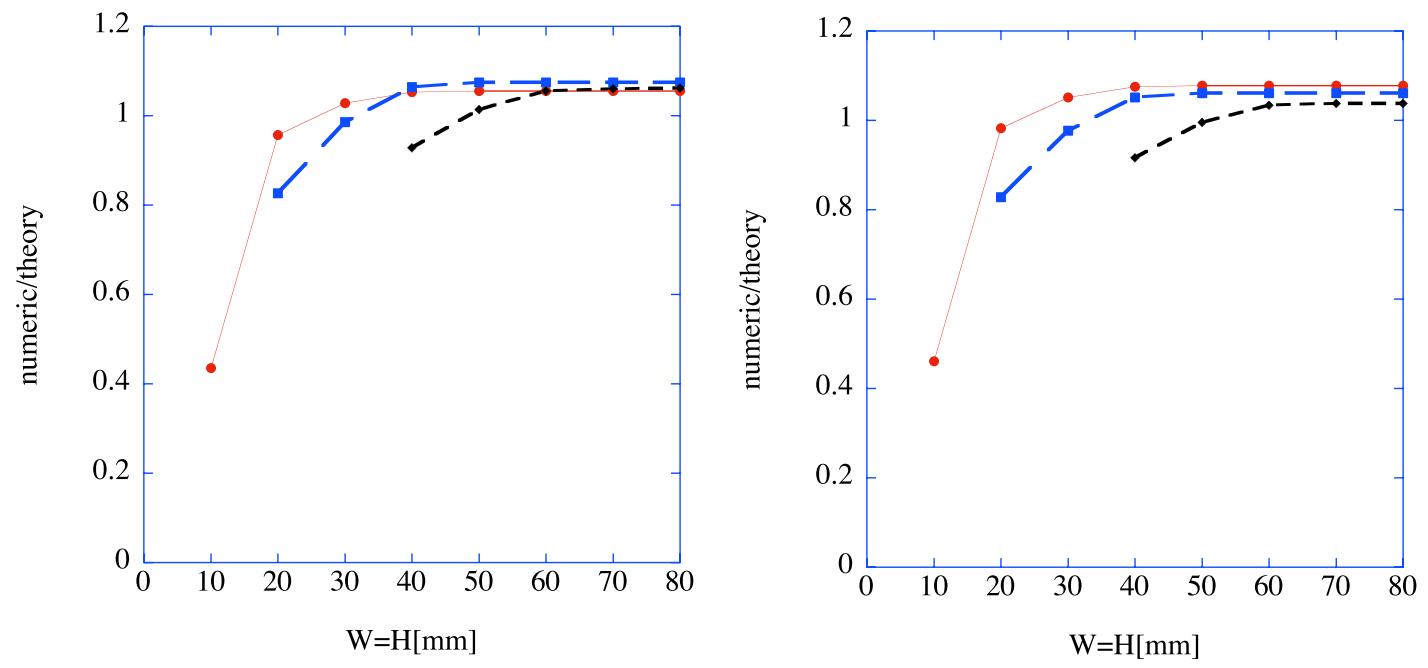

FIG. 6. (Color) The dependence of loss factor on the size of cavity. The vertical axis represents the ratio of the loss factor calculated by numerical calculation to that by the present theory [obtained from Eq. (17)]. The horizontal axis shows the value of $W=H$. The size of the beam $\sigma_{s}$ is chosen to be $1 \mathrm{~cm}$. The left and the right figures represent the $a=3.5$ and $a=5 \mathrm{~cm}$ cases, respectively. The red, blue, and black lines show $g=8, g=16$, and $g=32 \mathrm{~mm}$ cases, respectively. Roughly speaking, the loss factor of the cavity saturates to that of the gap when the size of the cavity $W=H$ exceeds twice the size of the gap. 
the saddle point method can be rigorously applicable in their theory. In this subsection, we verify the validity of their use of the saddle point method for the ultrarelativistic beam case by comparing with our results.

In the present theory, we can systematically deal with the fields not only inside the chamber, but also those outside the chamber. The radiation fields can be obtained in the same way as for the impedance:

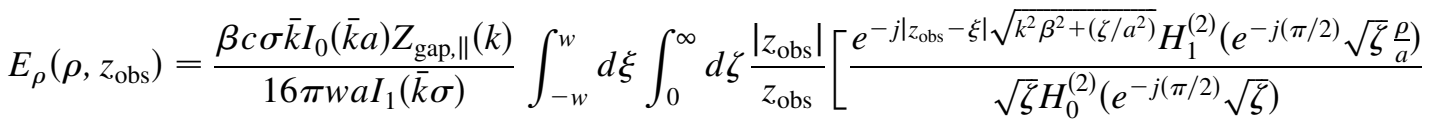

$$
\begin{aligned}
& \left.+\frac{e^{-j\left|z_{\mathrm{obs}}-\xi\right| \sqrt{k^{2} \beta^{2}-\left(\zeta / a^{2}\right)}} H_{1}^{(2)}\left(\sqrt{\zeta} \frac{\rho}{a}\right)}{j \sqrt{\zeta} H_{0}^{(2)}(\sqrt{\zeta})}\right] \\
& E_{z}\left(\rho, z_{\mathrm{obs}}\right)=-\frac{\beta c \sigma \bar{k} I_{0}(\bar{k} a) Z_{\mathrm{gap}, \|}(k)}{16 \pi w a^{2} I_{1}(\bar{k} \sigma)} \int_{-w}^{w} d \xi \int_{0}^{\infty} d \zeta\left[\frac{e^{-j\left|z_{\mathrm{obs}}-\xi\right| \sqrt{k^{2} \beta^{2}+\left(\zeta / a^{2}\right)}} H_{0}^{(2)}\left(e^{-(\pi / 2) j} \sqrt{\zeta} \frac{\rho}{a}\right)}{\sqrt{k^{2} \beta^{2}+\frac{\zeta}{a^{2}}} H_{0}^{(2)}\left(e^{-(\pi / 2) j} \sqrt{\zeta}\right)}\right. \\
& \left.+\frac{e^{-j\left|z_{\mathrm{obs}}-\xi\right| \sqrt{k^{2} \beta^{2}-\left(\zeta / a^{2}\right)}} H_{0}^{(2)}\left(\sqrt{\zeta} \frac{\rho}{a}\right)}{\sqrt{k^{2} \beta^{2}-\frac{\zeta}{a^{2}}} H_{0}^{(2)}(\sqrt{\zeta})}\right] \\
& H_{\theta}\left(\rho, z_{\mathrm{obs}}\right)=\frac{\beta^{2} \gamma c \sigma \bar{k}^{2} I_{0}(\bar{k} a) Z_{\mathrm{gap}, \|}}{16 \pi w a Z_{0} I_{1}(\bar{k} \sigma)} \int_{-w}^{w} d \xi \int_{0}^{\infty} d \zeta\left[\frac{e^{-j\left|z_{\mathrm{obs}}-\xi\right| \sqrt{k^{2} \beta^{2}+\left(\zeta / a^{2}\right)}} H_{1}^{(2)}\left(e^{-j(\pi / 2)} \sqrt{\zeta} \frac{\rho}{a}\right)}{\sqrt{\zeta} \sqrt{k^{2} \beta^{2}+\frac{\zeta}{a^{2}}} H_{0}^{(2)}\left(e^{-j(\pi / 2)} \sqrt{\zeta}\right)}\right. \\
& \left.+\frac{e^{-j\left|z_{\mathrm{obs}}-\xi\right| \sqrt{k^{2} \beta^{2}-\left(\zeta / a^{2}\right)}} H_{1}^{(2)}\left(\sqrt{\zeta} \frac{\rho}{a}\right)}{j \sqrt{\zeta} \sqrt{k^{2} \beta^{2}-\frac{\zeta}{a^{2}}} H_{0}^{(2)}(\sqrt{\zeta})}\right]
\end{aligned}
$$

Here we used Eqs. (D6) and (D7), and $\left(\rho, z_{\mathrm{obs}}\right)$ is the observing point. The first terms in Eqs. (18)-(20) decay quickly in the far field region and can be neglected. Since there is no pole of the Hankel function in $\zeta$-plane, we can change the path of integration, avoiding the cut due to the branch points at $\zeta=0$ and $\zeta=k^{2} \beta^{2} a^{2}$. Using the saddle point method, the radiation fields in the far zone, the second terms in Eqs. (18)-(20), can be approximated as (see Appendix E)

$$
\begin{gathered}
E_{\rho}\left(\rho, z_{\mathrm{obs}}\right) \simeq j \frac{\beta c \sigma \bar{k} I_{0}(\bar{k} a) Z_{\mathrm{gap}, \|}(k) e^{-j k \beta \bar{R}}}{2 \pi \bar{R} I_{1}(\bar{k} \sigma) H_{0}^{(2)}(k \beta a \sin \varphi)} \cot \varphi, \\
E_{z}\left(\rho, z_{\mathrm{obs}}\right) \simeq \frac{\beta c \sigma \bar{k} I_{0}(\bar{k} a) Z_{\mathrm{gap}, \|}(k) e^{-j k \beta \bar{R}}}{j 2 \pi \bar{R} I_{1}(\bar{k} a) H_{0}^{(2)}(k \beta a \sin \varphi)}, \\
H_{\theta}\left(\rho, z_{\mathrm{obs}}\right) \simeq j \frac{\beta c \sigma \bar{k} I_{0}(\bar{k} a) Z_{\mathrm{gap}, \|} e^{-j k \beta \bar{R}}}{2 \pi \bar{R} Z_{0} I_{1}(\bar{k} \sigma) H_{0}^{(2)}(k \beta a \sin \varphi) \sin \varphi},
\end{gathered}
$$

where $\rho=\bar{R} \sin \varphi$ and $z_{\mathrm{obs}}=\bar{R} \cos \varphi$. These expressions agree with those obtained by Silver and Saunders. Though they naively applied the saddle point approximation on their complex plane with a cut, their final results turn out to be correct [6].

\section{A FORMULA FOR TRANSVERSE IMPEDANCE WITH SPACE CHARGE EFFECTS}

A formula for the transverse impedance can be obtained similarly to that of the longitudinal impedance. We consider the situation where a beam is traveling in the chamber with the charge distribution of the following azimuthal dependence: $j_{z}=q \beta c \delta\left(\rho-r_{b}\right) \cos \theta e^{-j k z} / \pi r_{b}$.

Fields inside the chamber are described in Ref. [8] as (see Appendix A)

$$
\begin{aligned}
E_{z}= & i_{1}\left[\frac{j k}{\gamma^{2}} \frac{c Z_{0}}{\pi}\left(K_{1}\left(\bar{k} r_{b}\right)-K_{1}(\bar{k} a) \frac{I_{1}\left(\bar{k} r_{b}\right)}{I_{1}(\bar{k} a)}\right) I_{1}(\bar{k} \rho) \frac{e^{-j k z}}{r_{b}}\right. \\
& \left.+V_{2} \int_{-\infty}^{\infty} d h e^{-j h z} \frac{\sin h w}{h w} \frac{J_{1}(\Lambda \rho)}{J_{1}(\Lambda a)}\right] \cos \theta
\end{aligned}
$$

for $\rho<r_{b}$, and

$$
\begin{aligned}
E_{z}= & {\left[\frac{j k}{\gamma^{2}} \frac{c Z_{0}}{\pi} K_{1}(\bar{k} \rho) I_{1}\left(\bar{k} r_{b}\right) \frac{i_{1}}{r_{b}} e^{-j k z}\right.} \\
& \left.+\int_{-\infty}^{\infty} d h i_{1} A(h) e^{-j h z} \frac{J_{1}(\Lambda \rho)}{J_{1}(\Lambda a)}\right] \cos \theta,
\end{aligned}
$$




$$
\begin{aligned}
H_{\theta}= & {\left[c \beta \frac{\bar{k}}{2 \pi}\left[K_{0}(\bar{k} \rho)+K_{2}(\bar{k} \rho)\right] I_{1}\left(\bar{k} r_{b}\right) \frac{i_{1}}{r_{b}} e^{-j k z}\right.} \\
& -\int_{-\infty}^{\infty} d h e^{-j h z} \frac{j h}{\Lambda^{2}}\left(\frac{i_{1} B(h) J_{1}(\Lambda \rho)}{\rho J_{1}(\Lambda a)}\right. \\
& \left.\left.+\frac{k \beta}{Z_{0} h} i_{1} A(h) \frac{\frac{\partial J_{1}(\Lambda \rho)}{\partial \rho}}{J_{1}(\Lambda a)}\right)\right] \cos \theta \\
E_{\theta}= & H_{z}=\int_{-\infty}^{\infty} d h Z_{1} B(h) e^{-j h z} \frac{J_{1}(\Lambda \rho)}{J_{1}(\Lambda a)} \sin \theta \\
& \left.\times\left(i_{1} B(h) \frac{\frac{\partial J_{1}(\Lambda \rho)}{\partial \rho}}{J_{1}(\Lambda a)}+\frac{h}{Z_{0} k \beta \rho} i_{1} A(h) \frac{J_{1}(\Lambda \rho)}{J_{1}(\Lambda a)}\right)\right] \sin \theta
\end{aligned}
$$

for $\rho>r_{b}$, where $i_{1}=q r_{b}$.

Similarly to the longitudinal case, we first have to know fields outside the chamber to apply the field matching technique [7]. They are (see Appendix C)

$$
\begin{aligned}
E_{z}= & i_{1} V_{2} \int_{-\infty}^{\infty} d h \frac{H_{1}^{(2)}(\Lambda \rho)}{H_{1}^{(2)}(\Lambda a)} \frac{\sin h w}{h w} e^{-j h z} \cos \theta \\
H_{\theta}= & i_{1} V_{2} \int_{-\infty}^{\infty} d h\left(-j \frac{k \beta}{Z_{0}} \frac{\frac{\partial H_{1}^{(2)}(\Lambda \rho)}{\partial \rho}}{\Lambda^{2} H_{1}^{(2)}(\Lambda a)}\right. \\
& \left.+j \frac{h^{2}}{\rho} \frac{H_{1}^{(2)}(\Lambda \rho)}{Z_{0} k \beta a \Lambda^{2} \frac{\partial H_{1}^{(2)}(\Lambda a)}{\partial a}}\right) \frac{\sin h w}{h w} e^{-j h z} \cos \theta \\
& -i_{1} V_{1} \int_{-\infty}^{\infty} d h \frac{h}{\rho} \frac{H_{1}^{(2)}(\Lambda \rho)}{Z_{0} k \beta \frac{\partial H_{1}^{(2)}(\Lambda a)}{\partial a}} \frac{\sin h w}{h w} e^{-j h z} \cos \theta \\
H_{z}= & -\int_{-\infty}^{\infty} d h j \Lambda^{2}\left(i_{1} V_{1}-j \frac{i_{1} V_{2} h}{a \Lambda^{2}}\right) \frac{H_{1}^{(2)}(\Lambda \rho)}{Z_{0} k \beta \frac{\partial H_{1}^{(2)}(\Lambda a)}{\partial a}} \frac{\sin h w}{h w} \\
& \times e^{-j h z} \sin \theta, \\
E_{\theta}= & i_{1} V_{2} \int_{-\infty}^{\infty} d h\left(j \frac{h}{\rho \Lambda^{2}} \frac{H_{1}^{(2)}(\Lambda \rho)}{H_{1}^{(2)}(\Lambda a)}-j \frac{h}{a \Lambda^{2}} \frac{\frac{\partial H_{1}^{(2)}(\Lambda \rho)}{\partial \rho}}{\frac{\partial H_{1}^{(2)}(\Lambda a)}{\partial a}}\right) \\
& \times \frac{\sin h w}{h w} e^{-j h z} \sin \theta+i_{1} V_{1} \int_{-\infty}^{\infty} d h \frac{\frac{\partial H_{1}^{(2)}(\Lambda \rho)}{\partial \rho}}{\frac{\partial H_{1}^{(2)}(\Lambda a)}{\partial a}} \frac{\sin h w}{h w} \\
& \times e^{-j h z} \sin \theta .
\end{aligned}
$$

In the process of obtaining the above equation, we used the approximation that $V_{1}$ and $V_{2}$ are almost constant.

Since the fields given by Eqs. (25)-(28) and those by Eqs. (29)-(32) must be continuous at $\rho=a$, the gap voltage $V_{2}$ can be solved as

$$
V_{2}=\frac{j c Z_{0} I_{1}\left(\bar{k} r_{b}\right) e^{-j k z}}{\pi k a r_{b} I_{1}(\bar{k} a)\left(P-\frac{P_{3}}{k^{2} \beta^{2} a^{2}}\right)},
$$

$$
P=\frac{1}{2 w} \int_{-\infty}^{\infty} d h \int_{-w}^{w} d \xi \frac{e^{-j h(z-\xi)}}{\Lambda^{2}}\left(\frac{\frac{\partial H_{1}^{(2)}(\Lambda a)}{\partial a}}{H_{1}^{(2)}(\Lambda a)}-\frac{\frac{\partial J_{1}(\Lambda a)}{\partial a}}{J_{1}(\Lambda a)}\right)
$$

$$
\begin{aligned}
P_{3}= & \frac{1}{2 w} \int_{-\infty}^{\infty} d h \int_{-w}^{w} d \xi e^{-j h(z-\xi)} \frac{h^{2}}{\Lambda^{2}} \\
& \times\left(\frac{H_{1}^{(2)}(\Lambda a)}{\frac{\partial H_{1}^{(2)}(\Lambda a)}{\partial a}}-\frac{J_{1}(\Lambda a)}{\frac{\partial J_{1}(\Lambda a)}{\partial a}}\right),
\end{aligned}
$$

where $z$ is the matching point of magnetic fields.

If we insert Eq. (33) into Eq. (24) and use the PanofskyWenzel theorem [9], we can finally obtain the expression for the transverse impedance as

$$
\begin{aligned}
Z_{\perp}= & \frac{R Z_{0} k}{j \gamma^{3} \beta r_{b}}\left(K_{1}\left(\bar{k} r_{b}\right)-K_{1}(\bar{k} a) \frac{I_{1}\left(\bar{k} r_{b}\right)}{I_{1}(\bar{k} a)}\right) \\
& -\frac{\pi V_{2}}{c \beta \gamma I_{1}(\bar{k} a)} \frac{\sin k w}{k w}
\end{aligned}
$$

where $R=\mathcal{C} / 2 \pi$ is the average radius of the ring. The first term of Eq. (36) represents the transverse space charge impedance for the nonrelativistic beam (see Appendix B) [8], while the second term gives the transverse gap impedance $Z_{\perp \text {,gap. }}$ Using Eqs. (D1), (D2), (D4), and (D5), we can rewrite $Z_{\perp \text {,gap }}$ as

$$
Z_{\perp, \text { gap }}=-\frac{j Z_{0} \sin k w I_{1}\left(\bar{k} r_{b}\right) e^{-j k z}}{\beta \gamma r_{b} a k^{2} w I_{1}^{2}(\bar{k} a)\left(Y_{\text {pole }}^{\prime}+Y_{\text {cut }}^{\prime}\right)},
$$




$$
\begin{gathered}
Y_{\text {pole }}^{\prime}=\sum_{s=1}^{\infty}\left[-\frac{\pi a\left(2-e^{-j\left(b_{1, s} / w a\right)(z+w)}-e^{j\left(b_{1, s} / a\right)(z-w)}\right)}{w b_{1, s}^{2}}+\frac{\pi a J_{1}\left(j_{1, s}^{\prime}\right)\left(2-e^{-j\left(b_{1, s}^{\prime} / w\right)(z+w)}-e^{j\left(b_{1, s}^{\prime} / a\right)(z-w)}\right)}{k^{2} \beta^{2} a^{2} w j_{1, s}^{\prime 2} J_{1}^{\prime \prime}\left(j_{1, s}^{\prime}\right)}\right] \\
-\frac{\pi a H_{1}^{(2)}\left(h_{1,0}^{\prime}\right)\left(2-e^{-j\left(d_{1,0}^{\prime} / w\right)(z+w)}-e^{j\left(d_{1,0}^{\prime} / a\right)(z-w)}\right)}{k^{2} \beta^{2} a^{2} w h_{1,0}^{\prime 2} H_{1}^{\prime \prime(2)}\left(h_{1,0}^{\prime}\right)}+\frac{\pi a\left(2-e^{-j k \beta(z+w)}-e^{j k \beta(z-w)}\right)}{w k^{2} \beta^{2} a^{2}}\left(\frac{H_{1}^{(2)}\left(h_{1,0}^{\prime}\right)}{h_{1,0}^{\prime 2} H_{1}^{\prime \prime(2)}\left(h_{1,0}^{\prime}\right)}-\frac{1}{2}\right) \\
Y_{\mathrm{cut}}^{\prime}=-\frac{1}{\pi a w} \int_{0}^{\infty} d \zeta \frac{\left(2-e^{-j(z+w) \sqrt{k^{2} \beta^{2}+\left(\zeta / a^{2}\right)}}-e^{\left.j(z-w) \sqrt{k^{2} \beta^{2}+\left(\zeta / a^{2}\right)}\right)}\right.}{\zeta\left(k^{2} \beta^{2}+\frac{\zeta}{\left.a^{2}\right)} H_{1}^{(1)}\left(e^{j(\pi / 2)} \sqrt{\zeta}\right) H_{1}^{(2)}\left(e^{j(\pi / 2)} \sqrt{\zeta}\right)\right.} \\
+\int_{0}^{\infty} d \zeta \frac{\left(e^{-j(z+w) k \beta}+e^{j(z-w) k \beta}-e^{-j(z+w)} \sqrt{k^{2} \beta^{2}+\left(\zeta / a^{2}\right)}-e^{j(z-w)} \sqrt{k^{2} \beta^{2}+\left(\zeta / a^{2}\right)}\right)}{k^{2} \beta^{2} a \pi w \zeta^{2} H_{1}^{(1)}\left(e^{j(\pi / 2)} \sqrt{\zeta}\right) H_{1}^{(2)}\left(e^{j(\pi / 2)} \sqrt{\zeta}\right)} \\
\simeq 4 \tan ^{-1} \frac{1}{\sqrt{2 j k w}}+\frac{-2+4 \sqrt{1+2 j k \beta w} \sinh ^{-1} \frac{e^{-j(\pi / 4)}}{\sqrt{2 k \beta w}}+e^{-j(z+w) k \beta}+e^{j(z-w) k \beta}}{k^{2} \beta^{2} a^{2} \sqrt{1+2 j k \beta w}}
\end{gathered}
$$

where $\quad b_{1, s}=\sqrt{k^{2} \beta^{2} a^{2}-j_{1, s}^{2}}, \quad b_{1, s}^{\prime}=\sqrt{k^{2} \beta^{2} a^{2}-j_{1, s}^{\prime 2}}$, $d_{1,0}^{\prime}=\sqrt{k^{2} \beta^{2} a^{2}-h_{1,0}^{\prime 2}}, j_{n, s}$ are the sth zeros of $J_{n}(z), j_{1, s}^{\prime}$ are the sth zeros of $J_{1}^{\prime}(z)$ and $h_{1,0}^{\prime}=0.501184+$ $j 0.643545$ : the 0 th zero of $H_{1}^{\prime(2)}(z)$ (the differential of the Hankel function of the second kind). We should notice that $b_{1, s}$

approaches $-j \sqrt{j_{1, s}^{2}-k^{2} \beta^{2} a^{2}}$ for $j_{1, s}^{2}>k^{2} \beta^{2} a^{2}$ and $b_{1, s}^{\prime}$ approaches $-j \sqrt{j_{1, s}^{\prime 2}-k^{2} \beta^{2} a^{2}}$ for $j_{1, s}^{\prime 2}>k^{2} \beta^{2} a^{2}$.

Again, we reach the equation where the integration over $\zeta$ is just straightforward for numerical calculations, since there is no singular point along the integration path.

Let us check the accuracy of Eq. (37) for the transverse impedance again by comparing with the ABCI result. We

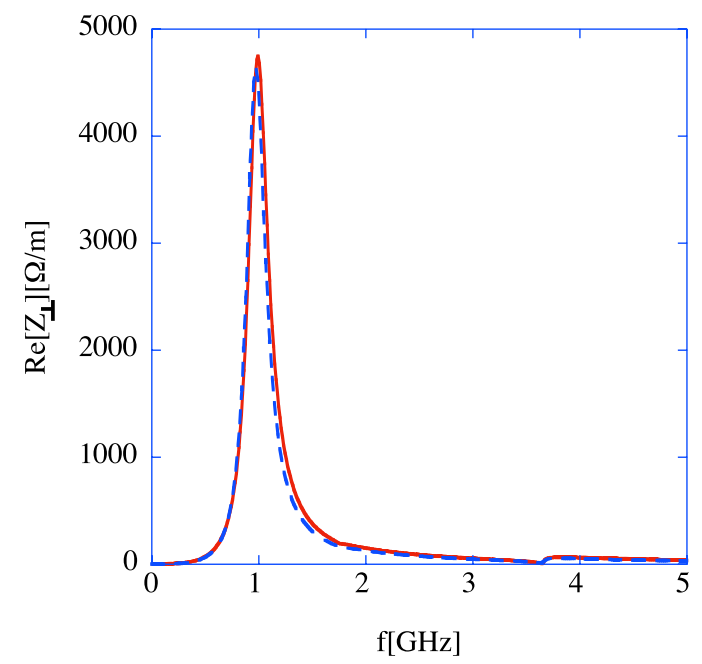

use the same set of parameters as for the longitudinal impedance case (namely, the chamber radius $a=50 \mathrm{~mm}$ and the half width of gap $w=4 \mathrm{~mm}$ ). Figure 7 shows the comparison results of the real (left) and imaginary (right) parts of the transverse impedance, respectively. The present theory and the ABCI result agree very well. Both show that the correct behavior of the impedance in high frequency, namely, it decreases in proportion to $k^{-3 / 2}$, as predicted by the diffraction theory. The theory also accurately produces the behavior of the impedance near the cutoff frequency, around $3.7 \mathrm{GHz}$ in Fig. 7.

It is interesting to point out that there is a large resonance peak at $1 \mathrm{GHz}$, which corresponds to the frequency where the wavelength is equal to the chamber circumference. This mode resonates along the aperture of the chamber,

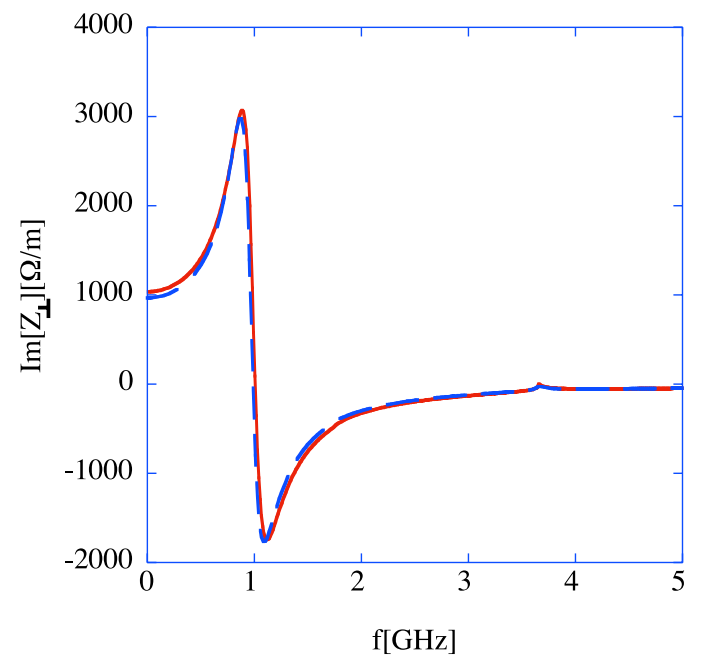

FIG. 7. (Color) Comparison of the real (left) and imaginary (right) parts of the transverse impedance, calculated by the present theory (red curves) and $\mathrm{ABCI}$ (blue curves), respectively. We used the same parameters as the longitudinal case. Namely, $\gamma=1000, a=5 \mathrm{~cm}$, $w=4 \mathrm{~mm}$, and $z=0 \mathrm{~m}$. The large resonance peak can be noticed at $1 \mathrm{GHz}$, which corresponds to the frequency where the wavelength is equal to the chamber circumference. 

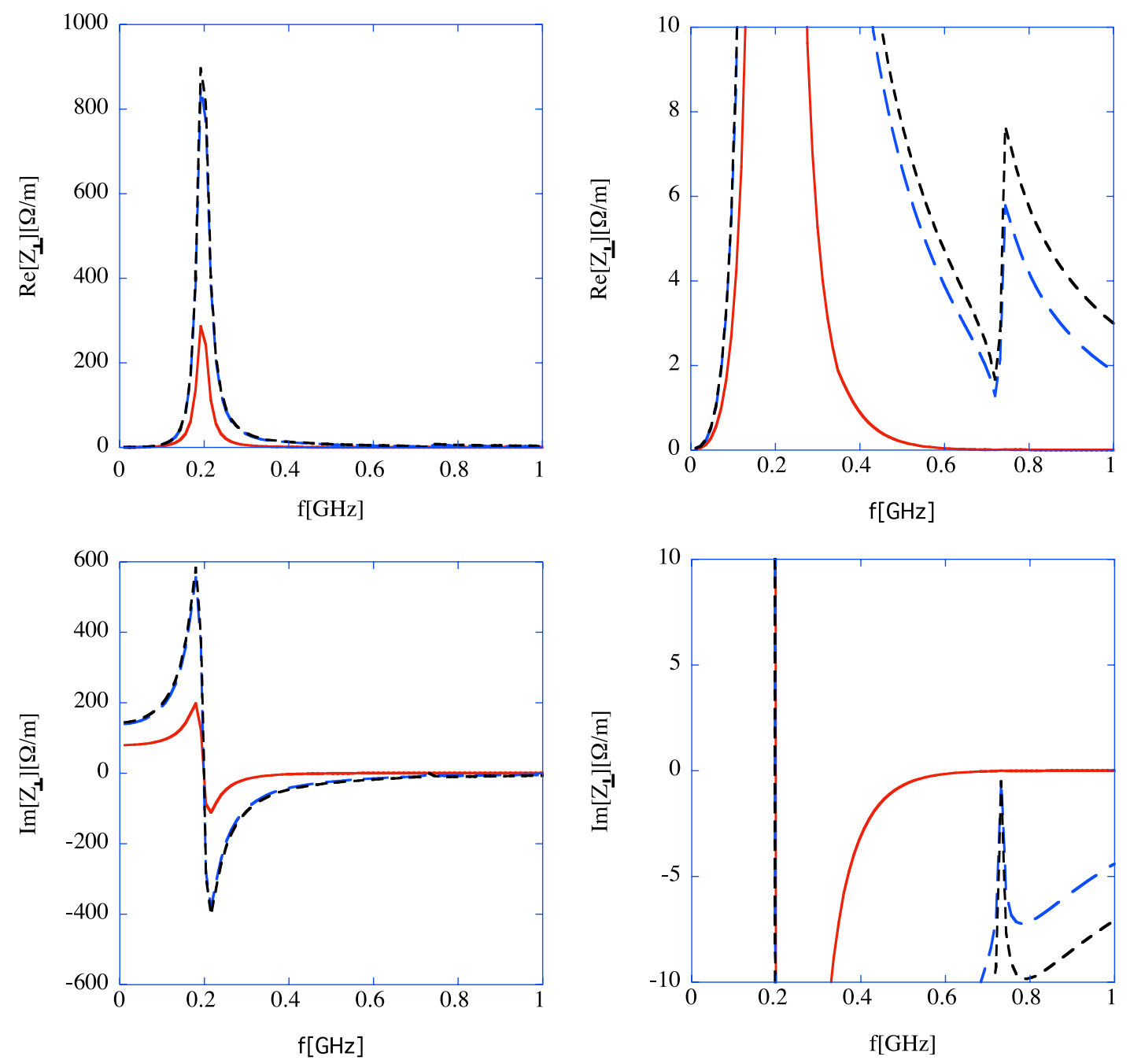

FIG. 8. (Color) The dependence of transverse impedance on Lorentz factor $\gamma$. We assume the chamber with large radius $a=250 \mathrm{~mm}$. The red, blue, and black curves represent $\gamma=1.2, \gamma=4$, and $\gamma=1000$ cases, respectively. The top and the bottom figures show the real and the imaginary parts of transverse impedance, respectively. The results for the nonrelativistic beam are smaller than those for the relativistic one.

and spreads out to the outside of chamber by about $30 \mathrm{~cm}$. This resonance gives a dominant contribution to the transverse impedance.

Our formalism can also deal with the transverse impedance for a nonrelativistic beam, just like the longitudinal impedance case. Figure 8 shows the dependence of transverse impedance on Lorentz factor $\gamma$ for the same chamber size $a=250 \mathrm{~mm}$ as in the longitudinal impedance case. It can be seen that the impedance for a nonrelativistic beam with $\gamma$ of 1.2 is indeed smaller than for a relativistic beam with $\gamma$ larger than several. It can also be identified that the modulation of impedance around the cutoff frequencies is more enhanced for a larger $\gamma$.

\section{SUMMARY}

We have presented the formulas for the longitudinal and transverse impedances due to a gap in the beam chamber.
In this process, we derived the complete solutions of electromagnetic fields effective in the entire region, including the inside and the outside of the chamber, in a form that they can be easily numerically evaluated. To our knowledge, this is the first time that such complete solutions have been obtained not only in the accelerator theory but also in the antenna theory. Particularly, the near field solution outside of the chamber needed the very careful treatment of Hankel functions in the integrations that have singular cuts and poles. The final integral expressions of the impedances are suitable for numerical calculations, because there is no singular point on the middle of the path of integration. The newly developed technique can provide new methods of solutions of electromagnetic fields also for a rather broad class of structures such as cavities. The numerical results of impedances are consistent with the $\mathrm{ABCI}$ results and their behavior in high frequency agrees well with the prediction of the diffraction theory. The most 
astonishing achievement is, however, that our theory can accurately reproduce the behavior of the impedance near and above the cutoff frequencies. Furthermore, our theory is applicable even to the impedances for nonrelativistic beams.

We made the conjecture that the short-range wake potential or the broadband impedance of a small cavitylike structure is almost determined by the gap size and the chamber radius. We investigated this conjecture by evaluating the loss factor and found that the loss factor of the cavitylike structure indeed agrees well with that of the gap if the size of the cavity is at least twice larger than the gap size. In other words, the broadband impedance of the small cavitylike structure can be estimated from the gap size and the chamber radius only, regardless of the exact shape of the structure.

We have the new discovery in the transverse impedance of a gap: there is a large resonance peak at the frequency where the wavelength is equal to the chamber circumference. It has been believed that a gap in the chamber or a ceramic break will increase the longitudinal impedance, but not the transverse one, and that the transverse impedance is increased only at very high frequency, around the pipe cutoff frequency, typically between 1 and $2 \mathrm{GHz}$. In reality, however, the transverse impedance is rather small at every frequency, except this resonance region. This resonance peak appears around $1-2 \mathrm{GHz}$ in most of cases, and we should be careful to design a ceramic break so that this transverse mode will not interfere with nearby devices such beam position monitors.

\section{ACKNOWLEDGMENTS}

The authors thank K. Yokoya, K. Oide, K. Ohmi, T. Toyama, and A. Ueno for discussions. The authors also thank the members of the J-PARC project at JAEA/ KEK.

\section{APPENDIX A: SOLUTIONS INSIDE THE CHAMBER}

The Maxwell equations can be written as wave equations. Assuming that electromagnetic fields have time dependency of $e^{j \omega t}$, they become Helmholtz equations:

$$
\begin{gathered}
\left(\Delta+k^{2} \beta^{2}\right) \vec{E}=j k \beta Z_{0} \vec{j}+\vec{\nabla}\left(c Z_{0} \bar{\rho}\right), \\
\left(\Delta+k^{2} \beta^{2}\right) \vec{H}=-\vec{\nabla} \times \vec{j} .
\end{gathered}
$$

tures, the wave equation for the longitudinal component of the electric and magnetic field contains no transverse field component and is decoupled from it. For the longitudinal field, there is a source term $c Z_{0} \partial \bar{\rho} / \partial z+j k \beta Z_{0} j_{z}$, while the $z$-component of $\vec{\nabla} \times \vec{j}$ vanishes for particles with the longitudinal velocity only.

We assume that a macroparticle with charge $q$ travels along the pipe at the constant radial offset position $\rho=$ $r_{b}, \theta=\theta_{b}$ with velocity $\beta c$ in the longitudinal direction. Then, the charge density is expressed as

$$
\begin{gathered}
\bar{\rho}=\frac{i_{m}}{r_{b}^{1+m}} \delta\left(\rho-r_{b}\right) \delta_{p}\left(\theta-\theta_{b}\right) \delta(z-\beta c t) \\
=\sum_{m=0}^{\infty} \int \frac{d k}{2 \pi} i_{m} \rho_{m}, \\
\rho_{m}=\frac{1}{\pi r_{b}^{1+m}\left(1+\delta_{m 0}\right)} \delta\left(\rho-r_{b}\right) \cos m\left(\theta-\theta_{b}\right) e^{-j k(z-\beta c t)}, \\
i_{m}=q r_{b}^{m},
\end{gathered}
$$

where $\delta(x)$ is the $\delta$-function, $\delta_{p}(\theta)$ is the periodic $\delta$-function, and $\delta_{m, n}$ is Kronecker $\delta$. Since the general solution of Maxwell equations is obtained by the superposition of those for $i_{m} \rho_{m}$, we choose $i_{m} \rho_{m}$ as the source term. Let us define the source field specified with subscript $S$ as the solution which satisfies the Maxwell equations with $\rho_{m}, \vec{j}_{m}$ and vanishes at $\rho \rightarrow \infty$. It is given by

$$
H_{\rho}^{S}=E_{\theta}^{S}=H_{z}^{S}=0,
$$

$$
E_{z}^{S}= \begin{cases}\frac{j k c Z_{0} I_{0}\left(\bar{k} r_{b}\right)}{2 \pi \gamma^{2}} K_{0}(\bar{k} \rho) e^{-j k z} & \text { for } \rho>r_{b} \\ \frac{j k c Z_{0} K_{0}\left(\bar{k} r_{b}\right)}{2 \pi \gamma^{2}} I_{0}(\bar{k} \rho) e^{-j k z} & \text { for } r_{b}>\rho\end{cases}
$$

$$
\frac{\beta}{Z_{0}} E_{\rho}^{S}=H_{\theta}^{S}= \begin{cases}\frac{\beta k c I_{0}\left(\bar{k}_{b}\right)}{2 \pi \gamma} K_{1}(\bar{k} \rho) e^{-j k z} & \text { for } \rho>r_{b} \\ -\frac{\beta k c K_{0}\left(\bar{k} r_{b}\right)}{2 \pi \gamma} I_{1}(\bar{k} \rho) e^{-j k z} & \text { for } r_{b}>\rho\end{cases}
$$

for $m=0$, and

$$
H_{z}^{S}=0
$$

$$
E_{z}^{S}= \begin{cases}\frac{j k c Z_{0} I_{m}\left(\bar{k} r_{b}\right)}{\pi r_{b}^{m} \gamma^{2}} K_{m}(\bar{k} \rho) \cos m\left(\theta-\theta_{b}\right) e^{-j k z} & \text { for } \rho>r_{b}, \\ \frac{j k c Z_{0} K_{m}\left(\bar{k} r_{b}\right)}{\pi r_{b}^{m} \gamma^{2}} I_{m}(\bar{k} \rho) \cos m\left(\theta-\theta_{b}\right) e^{-j k z} & \text { for } r_{b}>\rho,\end{cases}
$$

In the cylindrical coordinates for axially symmetric struc-

$$
-\frac{Z_{0}}{\beta} H_{\rho}^{S}=E_{\theta}^{S}= \begin{cases}\frac{m c Z_{0} I_{m}\left(\bar{k} r_{b}\right)}{\rho \pi r_{b}^{m}} K_{m}(\bar{k} \rho) \sin m\left(\theta-\theta_{b}\right) e^{-j k z} & \text { for } \rho>r_{b}, \\ \frac{m c Z_{0} K_{m}\left(\bar{k} r_{b}\right)}{\rho \pi r_{b}^{m}} I_{m}(\bar{k} \rho) \sin m\left(\theta-\theta_{b}\right) e^{-j k z} & \text { for } r_{b}>\rho,\end{cases}
$$




$$
\frac{\beta}{Z_{0}} E_{\rho}^{S}=H_{\theta}^{S}= \begin{cases}\frac{\beta k c I_{m}\left(\bar{k} r_{b}\right)}{2 \pi r_{b}^{m} \gamma}\left[K_{m-1}(\bar{k} \rho)+K_{m+1}(\bar{k} \rho)\right] \cos m\left(\theta-\theta_{b}\right) e^{-j k z} & \text { for } \rho>r_{b}, \\ -\frac{\beta k c K_{m}\left(\bar{k} r_{b}\right)}{2 \pi r_{b}^{m} \gamma}\left[I_{m-1}(\bar{k} \rho)+I_{m+1}(\bar{k} \rho)\right] \cos m\left(\theta-\theta_{b}\right) e^{-j k z} & \text { for } r_{b}>\rho,\end{cases}
$$

for $m>0$, where $K_{m}(z)$ and $I_{m}(z)$ are the modified Bessel functions, respectively, $k=2 \pi f / \beta c$ and $\bar{k}=k / \gamma$.

General solutions (especially $E_{z}, E_{\rho}, H_{\theta}$ ) inside the chamber for $m=0$ are expressed as

$$
\begin{gathered}
E_{z}=E_{z}^{S}+\int_{-\infty}^{\infty} d h A(h) e^{-j h z} \frac{J_{0}(\Lambda \rho)}{J_{0}(\Lambda a)}, \\
\frac{\beta}{Z_{0}} E_{\rho}=H_{\theta}=H_{\theta}^{S}+\frac{j k \beta}{Z_{0}} \int_{-\infty}^{\infty} d h A(h) e^{-j h z} \frac{J_{1}(\Lambda \rho)}{\Lambda J_{0}(\Lambda a)},
\end{gathered}
$$

and those (especially $E_{z}, E_{\rho}, H_{\theta}, H_{z}$ ) for $m>0$ are

$$
\begin{aligned}
E_{z}= & i_{m}\left[E_{z}^{S}+\int_{-\infty}^{\infty} d h A(h) e^{-j h z} \frac{J_{m}(\Lambda \rho)}{J_{m}(\Lambda a)} \cos m\left(\theta-\theta_{b}\right)\right], \\
H_{\theta}= & i_{m}\left[H_{\theta}^{S}-\int_{-\infty}^{\infty} d h e^{-j h z} \frac{j h}{\Lambda^{2}}\left(\frac{m B(h) J_{m}(\Lambda \rho)}{\rho J_{m}(\Lambda a)}\right.\right. \\
& \left.\left.+\frac{k \beta}{Z_{0} h} A(h) \frac{\frac{\partial J_{m}(\Lambda \rho)}{\partial \rho}}{J_{m}(\Lambda a)}\right) \cos m\left(\theta-\theta_{b}\right)\right], \\
H_{z}= & \int_{-\infty}^{\infty} d h i_{m} B(h) e^{-j h z} \frac{J_{m}(\Lambda \rho)}{J_{m}(\Lambda a)} \sin m\left(\theta-\theta_{b}\right), \\
E_{\theta}= & i_{m}\left[E_{\theta}^{S}+\int_{-\infty}^{\infty} d h e^{-j h z} \frac{j k \beta Z_{0}}{\Lambda^{2}}\right. \\
& \times\left(B(h) \frac{\frac{\partial J_{m}(\Lambda \rho)}{\partial \rho}}{J_{m}(\Lambda a)}+\frac{m h}{Z_{0} k \beta \rho} A(h) \frac{J_{m}(\Lambda \rho)}{J_{m}(\Lambda a)}\right) \\
& \left.\times \sin m\left(\theta-\theta_{b}\right)\right],
\end{aligned}
$$

where $J_{m}(z)$ is the Bessel function and $\Lambda=\sqrt{k^{2} \beta^{2}-h^{2}}$.

\section{APPENDIX B: SPACE CHARGE IMPEDANCES FOR A PERFECTLY CONDUCTIVE CHAMBER}

When a beam with the current density $j_{z}=\beta c[1-$ $\Theta(\rho-\sigma)] e^{-j k z} /\left(\pi \sigma^{2}\right)$, where $\Theta(x)$ is the step function, passes through the chamber, the source field (especially $E_{z}$ ) is expressed as

$$
\begin{aligned}
& E_{z}^{S}= \frac{j k c Z_{0} e^{-j k z}}{\pi \sigma^{2} \gamma^{2}}\left(\int_{0}^{\rho} d r_{b} r_{b} I_{0}\left(\bar{k} r_{b}\right) K_{0}(\bar{k} \rho)\right. \\
&\left.+\int_{\rho}^{\sigma} d r_{b} r_{b} K_{0}\left(\bar{k} r_{b}\right) I_{0}(\bar{k} \rho)\right) \\
&= \frac{j c Z_{0}}{\pi \gamma \sigma^{2}}\left(\frac{1}{\bar{k}}-\sigma I_{0}(\bar{k} \rho) K_{1}(\bar{k} \sigma)\right) e^{-j k z} \quad \text { for } \rho \leq \sigma, \\
& E_{z}^{S}=\frac{j k c Z_{0} e^{-j k z}}{\pi \sigma^{2} \gamma^{2}} \int_{0}^{\sigma} d r_{b} r_{b} I_{0}\left(\bar{k} r_{b}\right) K_{0}(\bar{k} \rho) \\
&=\frac{j c Z_{0}}{\pi \sigma \gamma} I_{1}(\bar{k} \sigma) K_{0}(\bar{k} \rho) e^{-j k z} \quad \text { for } \rho \geq \sigma .
\end{aligned}
$$

When the chamber is made of the perfectly conductive material, the longitudinal electric field inside the chamber is given by

$$
\begin{aligned}
E_{z}= & \frac{j c Z_{0}}{\pi \gamma \sigma^{2}}\left(\frac{1}{\bar{k}}-\sigma I_{0}(\bar{k} \rho) K_{1}(\bar{k} \sigma)\right) e^{-j k z} \\
& -\frac{j c Z_{0}}{\pi \sigma \gamma} I_{1}(\bar{k} \sigma) K_{0}(\bar{k} a) \frac{I_{0}(\bar{k} \rho)}{I_{0}(\bar{k} a)} e^{-j k z} \quad \text { for } \rho \leq \sigma, \\
E_{z}= & \frac{j c Z_{0}}{\pi \sigma \gamma} I_{1}(\bar{k} \sigma) K_{0}(\bar{k} \rho) e^{-j k z}-\frac{j c Z_{0}}{\pi \sigma \gamma} I_{1}(\bar{k} \sigma) K_{0}(\bar{k} a) \\
& \times \frac{I_{0}(\bar{k} \rho)}{I_{0}(\bar{k} a)} e^{-j k z} \quad \text { for } \rho \geq \sigma .
\end{aligned}
$$

The coupling impedance $Z_{\|}$is defined as the average of the longitudinal electric field (normalized by the beam current) over the beam cross section. Then, we obtain

$$
\begin{aligned}
Z_{\|}= & -\frac{j Z_{0}}{\beta \pi \sigma^{2} k}\left(1-\frac{2 K_{0}(\bar{k} a) I_{1}^{2}(\bar{k} \sigma)}{I_{0}(\bar{k} a)}\right. \\
& \left.-2 I_{1}(\bar{k} \sigma) K_{1}(\bar{k} \sigma)\right) \mathcal{C},
\end{aligned}
$$

where $\mathcal{C}$ is the ring circumference.

When a beam with the azimuthal dependency of $j_{z}=$ $q \beta c \delta\left(\rho-r_{b}\right) \cos \theta e^{-j k z} / \pi r_{b}$ is running inside the chamber, the longitudinal electric field is given by

$$
\begin{aligned}
E_{z}= & i_{1} \frac{j k c Z_{0} I_{1}\left(\bar{k} r_{b}\right)}{\pi r_{b} \gamma^{2}}\left(K_{1}(\bar{k} \rho)-K_{1}(\bar{k} a) \frac{I_{1}(\bar{k} \rho)}{I_{1}(\bar{k} a)}\right) \\
& \times \cos \left(\theta-\theta_{b}\right) e^{-j k z} \quad \text { for } \rho>r_{b},
\end{aligned}
$$




$$
\begin{aligned}
E_{z}= & i_{1} \frac{j k c Z_{0} I_{1}(\bar{k} \rho)}{\pi r_{b} \gamma^{2}}\left(K_{1}\left(\bar{k} r_{b}\right)-K_{1}(\bar{k} a) \frac{I_{1}\left(\bar{k} r_{b}\right)}{I_{1}(\bar{k} a)}\right) \\
& \times \cos \left(\theta-\theta_{b}\right) e^{-j k z} \quad \text { for } \rho<r_{b},
\end{aligned}
$$

where $i_{1}=q r_{b}$. Using the Panofsky-Wenzel theorem [9], we can obtain the transverse wake forces from the longitudinal electric fields. The transverse impedance is thus given by

$$
Z_{\perp}=\frac{R k Z_{0}}{j \beta r_{b} \gamma^{3}}\left(K_{1}\left(\bar{k} r_{b}\right)-\frac{I_{1}\left(\bar{k} r_{b}\right)}{I_{1}(\bar{k} a)} K_{1}(\bar{k} a)\right),
$$

where $R=\mathcal{C} / 2 \pi$ is the average radius of the ring.

\section{APPENDIX C: THE EXTERNAL FIELD PRODUCED BY A SLOT}

We construct the external field by superposition of basic sets of cylindrical waves. We only consider outward traveling waves (the Hankel function of the second kind). For any field component $E_{\alpha}(\rho, \theta, z)$, we can write

$$
E_{\alpha}(\rho, \theta, z)=\int_{-\infty}^{\infty} \mathcal{E}_{\alpha}(\rho, \theta, z) d h .
$$

Fourier series of electromagnetic fields are formally written as follows:

$$
\begin{aligned}
\mathcal{E}_{\rho}= & \sum_{n=-\infty}^{\infty}\left[-j h a_{n} \frac{\partial H_{n}^{(2)}(\Lambda \rho)}{\partial \rho}\right. \\
& \left.-\frac{n k \beta Z_{0}}{\rho} b_{n} H_{n}^{(2)}(\Lambda \rho)\right] e^{-j n \theta} e^{-j h z},
\end{aligned}
$$

$$
\begin{aligned}
\mathcal{E}_{\theta}= & \sum_{n=-\infty}^{\infty}\left[-\frac{n h}{\rho} a_{n} H_{n}^{(2)}(\Lambda \rho)\right. \\
+ & \left.j k \beta Z_{0} b_{n} \frac{\partial H_{n}^{(2)}(\Lambda \rho)}{\partial \rho}\right] e^{-j n \theta} e^{-j h z} \\
\mathcal{E}_{z}= & \sum_{n=-\infty}^{\infty} \Lambda^{2} a_{n} H_{n}^{(2)}(\Lambda \rho) e^{-j n \theta} e^{-j h z} \\
\mathcal{H}_{\rho}= & \sum_{n=-\infty}^{\infty}\left[\frac{n k \beta}{Z_{0} \rho} a_{n} H_{n}^{(2)}(\Lambda \rho)\right. \\
& \left.-j h b_{n} \frac{\partial H_{n}^{(2)}(\Lambda \rho)}{\partial \rho}\right] e^{-j n \theta} e^{-j h z}, \\
\mathcal{H}_{\theta}= & \sum_{n=-\infty}^{\infty}\left[-j \frac{k \beta}{Z_{0}} a_{n} \frac{\partial H_{n}^{(2)}(\Lambda \rho)}{\partial \rho}\right. \\
& \left.-\frac{n h}{\rho} b_{n} H_{n}^{(2)}(\Lambda \rho)\right] e^{-j n \theta} e^{-j h z}, \\
\mathcal{H}_{z}= & \sum_{n=-\infty}^{\infty} \Lambda^{2} b_{n} H_{n}^{(2)}(\Lambda \rho) e^{-j n \theta} e^{-j h z},
\end{aligned}
$$

where $H_{n}^{(2)}(z)$ is the Hankel function of the second kind.

The tangential electric field in the slot will have both $\theta$ and $z$ components in general. We express them using the prescribed functions $f_{1}(\theta, z)$ and $f_{2}(\theta, z)$ as

$$
\begin{aligned}
& f_{1}(\theta, z)= \begin{cases}E_{\theta}(a, \theta, z) & \text { on the slot }\left(\theta_{1} \leq \theta \leq \theta_{2} ; z_{1} \leq z \leq z_{2}\right) \\
0 & \text { outside the slot, }\end{cases} \\
& f_{2}(\theta, z)= \begin{cases}E_{z}(a, \theta, z) & \text { on the slot }\left(\theta_{1} \leq \theta \leq \theta_{2} ; z_{1} \leq z \leq z_{2}\right) \\
0 & \text { outside the slot. }\end{cases}
\end{aligned}
$$

The Fourier expansion coefficients are expressed as

$$
\begin{gathered}
a_{n}=\frac{1}{4 \pi^{2} \Lambda^{2} H_{n}^{(2)}(\Lambda a)} \int_{z_{1}}^{z_{2}} d \xi \int_{\theta_{1}(\xi)}^{\theta_{2}(\xi)} d \beta f_{2}(\beta, \xi) e^{j n \beta} e^{j h \xi} \\
b_{n}=-\frac{j n h}{4 \pi^{2} k \beta Z_{0} a \Lambda^{2}\left[\partial H_{n}^{(2)}(\Lambda a) / \partial a\right]} \int_{z_{1}}^{z_{2}} d \xi \int_{\theta_{1}(\xi)}^{\theta_{2}(\xi)} f_{2}(\beta, \xi) e^{j n \beta} e^{j h \xi} \\
-\frac{j}{4 \pi^{2} k \beta Z_{0}\left[\partial H_{n}^{(2)}(\Lambda a) / \partial a\right]} \int_{z_{1}}^{z_{2}} d \xi \int_{\theta_{1}(\xi)}^{\theta_{2}(\xi)} f_{1}(\beta, \xi) e^{j n \beta} e^{j h \xi}
\end{gathered}
$$

We consider the circumferential slot which goes all the way around the cylindrical chamber with the width of $2 w\left[z_{1}=-w, z_{2}=w, \theta_{1}(z)=0, \theta_{2}(z)=2 \pi\right]$ (which is much smaller than the chamber radius). Suppose that the tangential electric field in the slot has only the $z$ component, which is independent of $\theta$. Namely,

$$
f_{1}(\theta, z)=0 ; \quad f_{2}(\theta, z)=\frac{\tilde{V}}{2 w}
$$

Then, the nonzero coefficient is only for $n=0$ and is readily given by 


$$
a_{0}=\frac{\tilde{V} \sin h w / h w}{2 \pi \Lambda^{2} H_{0}^{(2)}(\Lambda a)} . \quad(\mathrm{C} 13) \quad f_{1}(\theta, z)=\frac{i_{1} V_{1} \pi}{w} ; \quad f_{2}(\theta, z)=\frac{i_{1} V_{2} \pi}{w} .
$$

The general expression of the fields can be written as

$$
\begin{gathered}
E_{z}=\frac{\tilde{V}}{2 \pi} \int_{-\infty}^{\infty} \frac{H_{0}^{(2)}(\Lambda \rho)}{H_{0}^{(2)}(\Lambda a)} \frac{\sin h w}{h w} e^{-j h z} d h, \\
H_{\theta}=j \frac{\beta k}{Z_{0}} \frac{\tilde{V}}{2 \pi} \int_{-\infty}^{\infty} \frac{H_{1}^{(2)}(\Lambda \rho)}{\Lambda H_{0}^{(2)}(\Lambda a)} \frac{\sin h w}{h w} e^{-j h z} d h .
\end{gathered}
$$

If the tangential electric field in the slot has both $z$ and $\theta$ components (especially $n=1$ case), we can get
Consequently, the only nonzero coefficients are

$$
\begin{gathered}
a_{ \pm 1}=\frac{i_{1} V_{2} \sin h w / h w}{2 \Lambda^{2} H_{ \pm 1}^{(2)}(\Lambda a)}, \\
b_{ \pm 1}= \pm\left(i_{1} V_{1}-j \frac{h i_{1} V_{2}}{a \Lambda^{2}}\right) \frac{\sin h w / h w}{2 k \beta Z_{0}\left[\partial H_{ \pm 1}^{(2)}(\Lambda a) / \partial a\right]} .
\end{gathered}
$$

Finally, we reach the general expression of the fields as

$$
\begin{gathered}
E_{z}=i_{1} V_{2} \int_{-\infty}^{\infty} d h \frac{H_{1}^{(2)}(\Lambda \rho)}{H_{1}^{(2)}(\Lambda a)} \frac{\sin h w}{h w} e^{-j h z} \cos \theta \\
H_{\theta}=i_{1} V_{2} \int_{-\infty}^{\infty} d h\left(-j \frac{k \beta}{Z_{0}} \frac{\frac{\partial H_{1}^{(2)}(\Lambda \rho)}{\partial \rho}}{\Lambda^{2} H_{1}^{(2)}(\Lambda a)}+j \frac{h^{2}}{\rho} \frac{H_{1}^{(2)}(\Lambda \rho)}{Z_{0} k \beta a \Lambda^{2} \frac{\partial H_{1}^{(2)}(\Lambda a)}{\partial a}}\right) \frac{\sin h w}{h w} e^{-j h z} \cos \theta \\
-i_{1} V_{1} \int_{-\infty}^{\infty} d h \frac{h}{\rho} \frac{H_{1}^{(2)}(\Lambda \rho)}{Z_{0} k \beta \frac{\partial H_{1}^{(2)}(\Lambda a)}{\partial a}} \frac{\sin h w}{h w} e^{-j h z} \cos \theta \\
H_{z}=-\int_{-\infty}^{\infty} d h j \Lambda^{2}\left(i_{1} V_{1}-j \frac{i_{1} V_{2} h}{a \Lambda^{2}}\right) \frac{H_{1}^{(2)}(\Lambda \rho)}{Z_{0} k \beta \frac{\partial H_{1}^{(2)}(\Lambda a)}{\partial a}} \frac{\sin h w}{h w} e^{-j h z} \sin \theta \\
E_{\theta}=i_{1} V_{2} \int_{-\infty}^{\infty} d h\left(j \frac{h}{\rho \Lambda^{2}} \frac{H_{1}^{(2)}(\Lambda \rho)}{H_{1}^{(2)}(\Lambda a)}-j \frac{h}{a \Lambda^{2}} \frac{\frac{\partial H_{1}^{(2)}(\Lambda \rho)}{\partial \rho}}{\frac{\partial H_{1}^{(2)}(\Lambda a)}{\partial a}}\right) \frac{\sin h w}{h w} e^{-j h z} \sin \theta+i_{1} V_{1} \int_{-\infty}^{\infty} d h \frac{\frac{\partial H_{1}^{(2)}(\Lambda \rho)}{\partial \rho}}{\frac{\partial H_{1}^{(2)}(\Lambda a)}{\partial a}} \frac{\sin h w}{h w} e^{-j h z} \sin \theta
\end{gathered}
$$

\section{APPENDIX D: EXPANSION FORMULAS OF THE BESSEL AND HANKEL FUNCTIONS}

The Bessel functions have the following relationships:

$$
\begin{gathered}
\frac{J_{n}^{\prime}(z)}{J_{n}(z)}=\frac{n}{z}+\sum_{k=1}^{\infty} \frac{2 z}{z^{2}-j_{n, k}^{2}}, \\
\frac{J_{1}(z)}{z J_{1}^{\prime}(z)}=\sum_{k=1}^{\infty} \frac{2}{z^{2}-j_{1, k}^{\prime 2}} \frac{J_{1}\left(j_{1, k}\right)}{J_{1}^{\prime \prime}\left(j_{1, k}^{\prime}\right)},
\end{gathered}
$$

where $j_{n, k}$ are the $k$ th zeros of $J_{n}(z)$ and $j_{1, k}^{\prime}$ are the $k$ th zeros of $J_{1}^{\prime}(z)$ [10].

Similar formulas can be obtained for the Hankel functions after some manipulations as follows:

$$
\frac{1}{\sqrt{z}} \frac{H_{1}^{(2)}(\sqrt{z})}{H_{0}^{(2)}(\sqrt{z})}=\int_{0}^{\infty} d \zeta \frac{2}{\pi^{2} \zeta(\zeta+z) H_{0}^{(1)}\left(e^{(\pi / 2) j} \sqrt{\zeta}\right) H_{0}^{(2)}\left(e^{(\pi / 2) j} \sqrt{\zeta}\right)}
$$




$$
\begin{gathered}
\frac{1}{\sqrt{z}} \frac{H_{1}^{\prime(2)}(\sqrt{z})}{H_{1}^{(2)}(\sqrt{z})}=-\frac{1}{z}-\int_{0}^{\infty} d \zeta \frac{2}{\pi^{2} \zeta(\zeta+z) H_{1}^{(1)}\left(e^{(\pi / 2) j} \sqrt{\zeta}\right) H_{1}^{(2)}\left(e^{(\pi / 2) j} \sqrt{\zeta}\right)} \\
\frac{1}{\sqrt{z}} \frac{H_{1}^{(2)}(\sqrt{z})}{H_{1}^{\prime(2)}(\sqrt{z})}=\frac{2 H_{1}^{(2)}\left(h_{1,0}^{\prime}\right)}{\left(z-h_{1,0}^{\prime 2}\right) H_{1}^{\prime \prime(2)}\left(h_{1,0}^{\prime}\right)}+\int_{0}^{\infty} d \zeta \frac{2}{\pi^{2} \zeta(\zeta+z) H_{1}^{(1)}\left(e^{(\pi / 2) j} \sqrt{\zeta}\right) H_{1}^{(2)}\left(e^{(\pi / 2) j} \sqrt{\zeta}\right)} \\
\frac{1}{\sqrt{z_{1}}} \frac{H_{1}^{(2)}\left(\sqrt{z_{1}} \alpha\right)}{H_{0}^{(2)}\left(\sqrt{z_{1}}\right)}=\frac{1}{2 \pi} \int_{0}^{\infty} d \zeta\left(\frac{H_{1}^{(2)}\left(e^{-(\pi / 2) j} \sqrt{\zeta} \alpha\right)}{\sqrt{\zeta}\left(\zeta+z_{1}\right) H_{0}^{(2)}\left(e^{-(\pi / 2) j} \sqrt{\zeta}\right)}-\frac{H_{1}^{(2)}(\sqrt{\zeta} \alpha)}{j \sqrt{\zeta}\left(\zeta-z_{1}\right) H_{0}^{(2)}(\sqrt{\zeta})}\right) \\
\frac{H_{0}^{(2)}\left(\sqrt{z_{1}} \alpha\right)}{H_{0}^{(2)}\left(\sqrt{z_{1}}\right)}=\frac{1}{2 \pi j} \int_{0}^{\infty} d \zeta\left(\frac{H_{0}^{(2)}\left(e^{-(\pi / 2) j} \sqrt{\zeta} \alpha\right)}{\left(\zeta+z_{1}\right) H_{0}^{(2)}\left(e^{-(\pi / 2) j} \sqrt{\zeta}\right)}-\frac{H_{0}^{(2)}(\sqrt{\zeta} \alpha)}{\left(\zeta-z_{1}\right) H_{0}^{(2)}(\sqrt{\zeta})}\right)
\end{gathered}
$$

where $\alpha>1, \quad-\pi<\arg \left(z_{1}\right)<0, \quad h_{1,0}^{\prime}=0.501184+$ $j 0.643545$ : the 0 th zero of $H_{1}^{\prime(2)}(z)$ (the differential of the Hankel function of the second kind) and $H_{\nu}^{(1)}(z)$ is the Hankel function of the first kind.

Let us prove the above formulas in the following way. Let us consider the following form of integral

$$
\frac{1}{2 \pi j} \oint \frac{F(\zeta)}{\sqrt{\zeta}(\zeta-z)} d \zeta
$$

First we take $F(\zeta)$ as

$$
F(\zeta)=\frac{H_{1}^{(2)}(\sqrt{\zeta})}{H_{0}^{(2)}(\sqrt{\zeta})},
$$

to prove Eq. (D3). We choose the path of the integral as shown in Fig. 9. The contribution of the integral from the

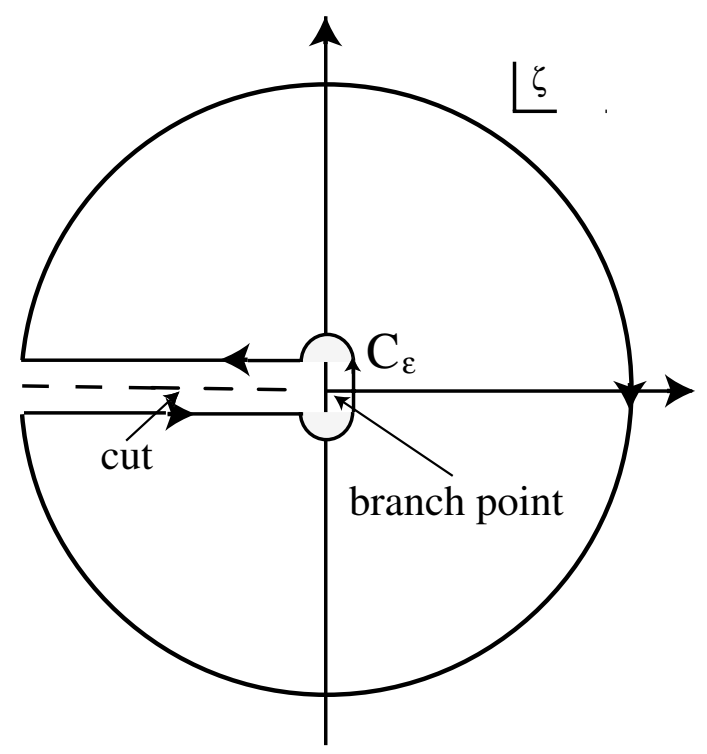

FIG. 9. The path of integration in Eq. (D8) in order to prove Eqs. (D3)-(D5). infinity vanishes since $F(\zeta)$ approaches 1 as $|\zeta| \rightarrow \infty$. The contribution from $C_{\epsilon}$ approaches zero as $\epsilon \rightarrow 0$. The contribution from the poles except $\zeta=z$ vanishes because the Hankel function $H_{0}^{(2)}(z)$ has no poles in $-\pi / 2<z<\pi / 2$, which corresponds to $-\pi<\zeta<\pi$. Then, the following relationship can be found:

$$
\begin{aligned}
\frac{H_{1}^{(2)}(\sqrt{z})}{\sqrt{z} H_{0}^{(2)}(\sqrt{z})}= & \frac{1}{2 \pi} \int_{0}^{\infty} \frac{d \zeta}{\sqrt{\zeta}(\zeta+z)} \\
& \times\left[\frac{H_{1}^{(2)}\left(e^{(\pi / 2) j} \sqrt{\zeta}\right)}{H_{0}^{(2)}\left(e^{(\pi / 2) j} \sqrt{\zeta}\right)}+\frac{H_{1}^{(2)}\left(e^{-(\pi / 2) j} \sqrt{\zeta}\right)}{H_{0}^{(2)}\left(e^{-(\pi / 2) j} \sqrt{\zeta}\right)}\right]
\end{aligned}
$$

We now derive Eq. (D3) by using the relationships

$$
\begin{gathered}
H_{0}^{(2)}\left(e^{-\pi j} z\right)=-H_{0}^{(1)}(z), \\
H_{1}^{(2)}\left(e^{-\pi j} z\right)=H_{1}^{(1)}(z), \\
H_{0}^{(1)}(z) H_{1}^{(2)}(z)-H_{1}^{(1)}(z) H_{0}^{(2)}(z)=\frac{4 j}{\pi z},
\end{gathered}
$$

which can be found in Ref. [11].

Similarly, in order to prove Eq. (D4), we take $F(\zeta)$ as

$$
F(\zeta)=\frac{H_{1}^{\prime(2)}(\sqrt{\zeta})}{H_{1}^{(2)}(\sqrt{\zeta})} .
$$

In this case, the contribution from the circle $C_{\epsilon}$ does not vanish. The following relationships can be found:

$$
\begin{aligned}
\frac{H_{1}^{\prime(2)}(\sqrt{z})}{\sqrt{z} H_{1}^{(2)}(\sqrt{z})}= & -\frac{1}{z}+\frac{1}{2 \pi} \int_{0}^{\infty} \frac{d \zeta}{\sqrt{\zeta}(\zeta+z)} \\
& \times\left[\frac{H_{1}^{\prime(2)}\left(e^{(\pi / 2) j} \sqrt{\zeta}\right)}{H_{1}^{(2)}\left(e^{(\pi / 2) j} \sqrt{\zeta}\right)}+\frac{H_{1}^{(2)}\left(e^{-(\pi / 2) j} \sqrt{\zeta}\right)}{H_{1}^{(2)}\left(e^{-(\pi / 2) j} \sqrt{\zeta}\right)}\right] .
\end{aligned}
$$



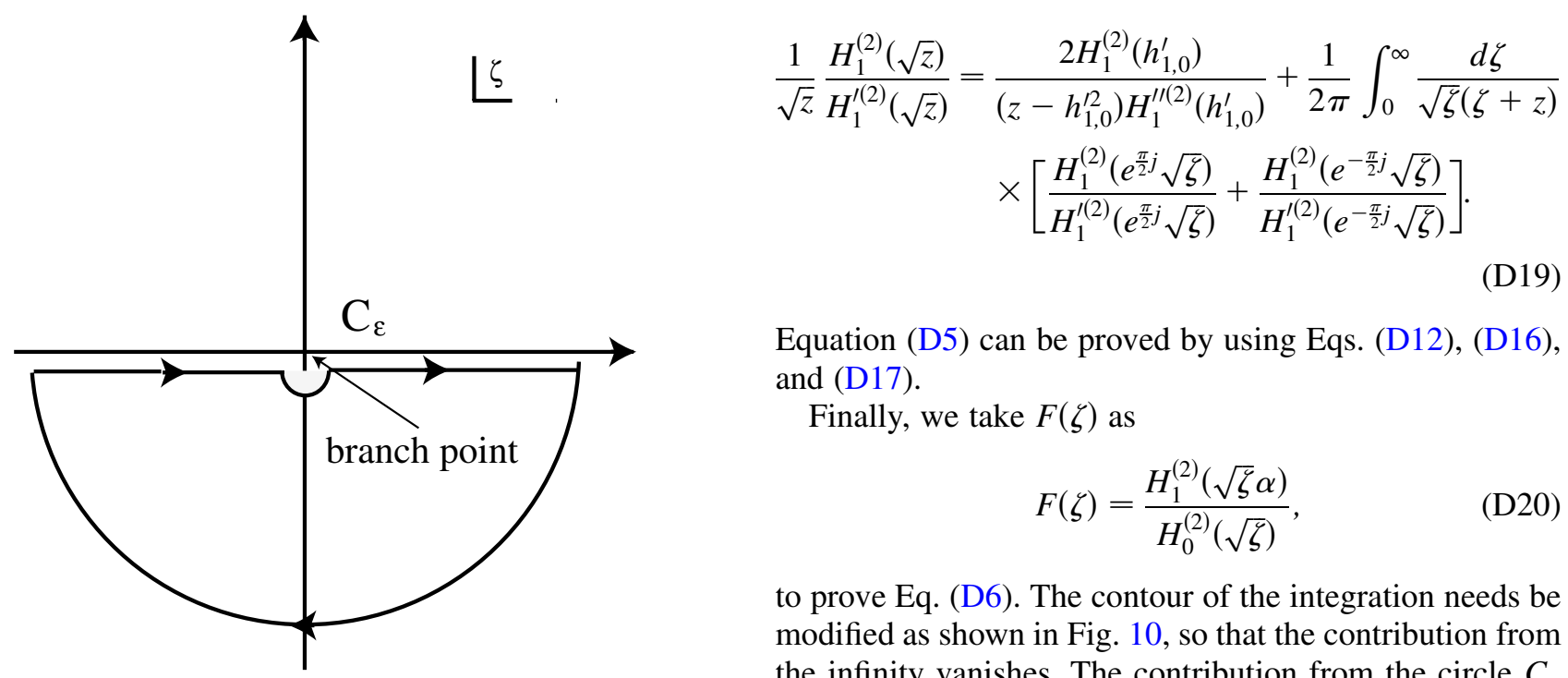

FIG. 10. The path of integration in Eq. (D8) in order to prove Eq. (D6).

We can derive Eq. (D4) using Eq. (D12) and the relationships

$$
\begin{gathered}
H_{1}^{\prime(2)}\left(e^{-\pi j} z\right)=-H_{1}^{\prime(1)}(z), \\
H_{1}^{(1)}(z) H_{1}^{\prime(2)}(z)-H_{1}^{\prime(1)}(z) H_{1}^{(2)}(z)=-\frac{4 j}{\pi z} .
\end{gathered}
$$

For proof of Eq. (D5), we take $F(\zeta)$ as

$$
F(\zeta)=\frac{H_{1}^{(2)}(\sqrt{\zeta})}{H_{1}^{\prime(2)}(\sqrt{\zeta})}
$$

The contribution from the poles of the Hankel functions does not vanish in this case, while that from the circle $C_{\epsilon}$ vanishes. Then, we can obtain the following relationships:
(D19)

Equation (D5) can be proved by using Eqs. (D12), (D16), and (D17).

Finally, we take $F(\zeta)$ as

$$
F(\zeta)=\frac{H_{1}^{(2)}(\sqrt{\zeta} \alpha)}{H_{0}^{(2)}(\sqrt{\zeta})}
$$

to prove Eq. (D6). The contour of the integration needs be modified as shown in Fig. 10, so that the contribution from the infinity vanishes. The contribution from the circle $C_{\epsilon}$ approaches zero as $\epsilon \rightarrow 0$. The position $z\left(=z_{1}\right)$ lies on the lower half of the complex plane. Equation (D7) can be obtained by integrating Eq. (D6) with $\alpha$ from 1 to $\alpha$.

\section{APPENDIX E: THE FAR-ZONE FIELDS}

The general expression for the far-zone region is obtained by making use of the asymptotic form of the Hankel function and the saddle point method. The first terms in Eqs. (18)-(20) are negligibly small in this region and can be neglected. There is no pole of the Hankel function in the $\zeta$-plane. We can change the path of integration, avoiding the cut due to the branch points at $\zeta=0$ and $\zeta=k^{2} \beta^{2} a^{2}$. The asymptotic form of the Hankel function is expressed as

$$
H_{n}^{(2)}(z) \sim \sqrt{\frac{2}{\pi z}} e^{-j[z-(2 n+1) \pi / 4]},
$$

and then the second terms of Eqs. (18)-(20) are described as

$$
\begin{gathered}
E_{\rho}\left(\rho, z_{\mathrm{obs}}\right)=\frac{\left|z_{\mathrm{obs}}\right| Z_{\mathrm{gap}, \|}}{z_{\mathrm{obs}} \sqrt{2 a \rho}} \frac{\beta c \sigma \bar{k} I_{0}(\bar{k} a)}{4 \pi^{3 / 2} I_{1}(\bar{k} \sigma)} e^{j(3 / 4) \pi} \int_{0}^{\infty} d \zeta \frac{e^{\Phi(\zeta)}}{j \zeta^{3 / 4} H_{0}^{(2)}(\sqrt{\zeta})}, \\
E_{z}\left(\rho, z_{\mathrm{obs}}\right)=-\frac{\beta c \sigma \bar{k} I_{0}(\bar{k} a) Z_{\mathrm{gap}, \|}}{8 \pi a^{2} I_{1}(\bar{k} \sigma)} \sqrt{\frac{2 a}{\pi \rho}} e^{j(\pi / 4)} \int_{0}^{\infty} d \zeta \frac{e^{\Phi(\zeta)}}{\zeta^{1 / 4} \sqrt{k^{2} \beta^{2}-\frac{\zeta}{a^{2}}} H_{0}^{(2)}(\sqrt{\zeta})} \\
H_{\theta}\left(\rho, z_{\mathrm{obs}}\right)=\frac{k \beta Z_{\mathrm{gap}, \|}}{\sqrt{2 a \rho} Z_{0}} \frac{\beta c \sigma \bar{k} I_{0}(\bar{k} a)}{4 \pi^{3 / 2} I_{1}(\bar{k} \sigma)} e^{j(3 / 4) \pi} \int_{0}^{\infty} d \zeta \frac{e^{\Phi(\zeta)}}{j \zeta^{3 / 4} \sqrt{k^{2} \beta^{2}-\frac{\zeta}{a^{2}}} H_{0}^{(2)}(\sqrt{\zeta})}
\end{gathered}
$$

where

$$
\Phi(\zeta)=-j\left|z_{\mathrm{obs}}\right| \sqrt{k^{2} \beta^{2}-\frac{\zeta}{a^{2}}}-j \sqrt{\zeta} \frac{\rho}{a} .
$$

Since the saddle point is located at $\zeta=k^{2} \beta^{2} a^{2} \rho^{2} /\left(z_{\mathrm{obs}}^{2}+\rho^{2}\right)$, we can derive Eqs. (21)-(23). 
[1] For example, S. Sato et al., in Proceedings of the LINAC2006, Knoxville TN, 2006, p. 268, http://cern.ch/ AccelConf/106/PAPERS/TUP011.PDF.

[2] C. H. Papas, J. Appl. Phys. 20, 437 (1949), http://scitation. aip.org/getabs/servlet/GetabsServlet prog=normal\&id= JAPIAU000020000005000437000001 \& idtype $=$ cvips \& gifs=yes.

[3] K. Bane and M. Sands, Part. Accel. 25, 73 (1990), http:// www. slac. stanford .edu/pubs/slacpubs/4000/slac-pub4441.html.

[4] Y.H. Chin, KEK Report 2005-06, 2005, http://www-lib. kek.jp/cgi-bin/kiss_prepri.v8?KN=200524006\&OF=8.

[5] B. Zotter and F. Sacherer, in Proceedings of Theoretical Aspects of the Behaviour of Beams in Accelerators and Storage Rings, Erice, Sicily (CERN Report No. 77-13, 1977), p. 175, http://preprints.cern.ch/cgi-bin/setlink? base=cernrep\&categ=Yellow_Report\&id=77-13.

[6] S. Silver and W.K. Saunders, J. Appl. Phys. 21, 153 (1950), http://scitation.aip.org/getabs/servlet/ GetabsServlet ? prog = normal\&id=JAPIAU000021000002
$000153000001 \&$ idtype $=$ cvips $\&$ gifs $=$ yes.

[7] B. W. Zotter and S. A. Kheifets, Impedances and Wakes in High-Energy Particle Accelerators (World Scientific, Singapore, 1998), http://www.worldscibooks.com/ physics/3068.html.

[8] Y. Shobuda et al., in Proceedings of the Particle Accelerator Conference 2005, Knoxville, TN (IEEE, Piscataway, NJ, 2005), p. 1898, http://cern.ch/ AccelConf/p05/PAPERS/MPPP025.PDF.

[9] A. W. Chao, Physics of Collective Beam Instabilities in High Energy Accelerators (Wiley, New York, 1993), http://www.slac.stanford.edu/ achao/wileybook.html.

[10] R. L. Gluckstern and R. Li, Part. Accel. 29, 159 (1990), http://www-spires.slac.stanford.edu/spires/find/hep?key= 2140101.

[11] M. Abramowitz and I. Stegun, Handbook of Mathematical Functions-With Formulas, Graphs, and Mathematical Tables (Dover Publications, New York, 1974), http:// www.math.sfu.ca/ cbm/aands/. 